Original Research Paper

\title{
Future Medicine Services Robotics
}

\author{
${ }^{1}$ Relly Victoria V. Petrescu, ${ }^{2}$ Raffaella Aversa, ${ }^{2}$ Antonio Apicella and ${ }^{1}$ Florian Ion T. Petrescu \\ ${ }^{1}$ ARoTMM-IFToMM, Romanian Society of Robotics (SRR), \\ Bucharest Polytechnic University, Bucharest, (CE) Romania \\ ${ }^{2}$ Advanced Material Lab, \\ Department of Architecture and Industrial Design, Second University of Naples, Aversa (CE) Italy
}

\author{
Article history \\ Received: 20-11-2016 \\ Revised: 22-11-2016 \\ Accepted: 26-11-2016 \\ Corresponding Author: \\ Florian Ion T. Petrescu \\ Member of Romanian Society \\ of Robotics Committee, \\ Bucharest branch; \\ Vice President of ARoTMMB- \\ IFToMM \\ Emails: scipub02@gmail.com
}

\begin{abstract}
It is an entry technique in use recently, albeit in selected centers and represents a further step in the field of minimally invasive surgery. Basically it has the same indications but, at present, is reserved for selected patients. Compared to traditional video-assisted surgery presents some important differences. The surgeon is physically distant from the operative field and sits at a console, equipped with a monitor, from which, through a complex system, controls the movement of the robotic arms. These are fixed the various surgical instruments, tweezers, scissors, dissectors, that team shall present to the operating table to introduce into the cavity operative site. The use of mechanical arms has the advantage of allowing a three dimensional view an image with more stops and to make the most delicate maneuvers purposes and also because the tools are articulated to the distal end. The disadvantage is related to the times longer operative and the difficulty of determining the strength (as can happen in giving the right tension to a surgeon's knot). In the future it can be assumed that robotic surgery will allow, with the development of the experience, the spread of the equipment and improvement of telecommunication systems and data, to operate at ever greater distances. If you think that today, the space centers, you can operate the robots sent to the moon or farther away, it is not hard to believe that it will become usual to operate from side to side of the area, providing you with all the best and specific skills. The first surgical robot called da Vinci, in honor of Leonardo da Vinci, was developed in Silicon Valley by Intuitive Surgical and in 2000 he obtained the authorization of the American Food and Drug Administration (FDA) for use in laparoscopic surgery. The present paper wishes to show briefly several models of the main robots placed in the service of human medicine.
\end{abstract}

Keywords: Robotics, Mechatronics, Biomimetic, Medical Robotics, Medicine Services Robotics, Surgery Robotics Assisted, Creativity, Evolutionary Design, Sustainable Development, Innovation, Engineering Design, Industrial Design, Mechanical Design, Mechanical Transmissions

\section{Introduction}

It is an entry technique in use recently, albeit in selected centers and represents a further step in the field of minimally invasive surgery. Basically it has the same indications but, at present, is reserved for selected patients. Compared to traditional video-assisted surgery presents some important differences. The surgeon is physically distant from the operative field and sits at a console, equipped with a monitor, from which, through a complex system, controls the movement of the robotic arms. These are fixed the various surgical instruments, tweezers, scissors, dissectors, that team shall present to the operating table to introduce into the cavity operative site. The use of mechanical arms has the advantage of allowing a three dimensional view an image with more stops and to make the most delicate maneuvers purposes and also because the tools are articulated to the distal end. The disadvantage is related to the times longer operative and the difficulty of determining the strength (as can happen in giving the right tension to a surgeon's knot), (Chirurgia robotica, 
Da Wikipedia). In the future it can be assumed that robotic surgery will allow, with the development of the experience, the spread of the equipment and improvement of telecommunication systems and data, to operate at ever greater distances. If you think that today, the space centers, you can operate the robots sent to the moon or farther away, it is not hard to believe that it will become usual to operate from side to side of the area, providing you with all the best and specific skills. In Grosseto (center of excellence), there is a school of robotics founded by Prof. Pier Cristoforo Giulianotti surgery, which trains general surgeons, urologists and gynecologists from the national and international territory (Fig. 1).

Thanks to the fruitful collaboration, the Urology of Grosseto, the first in central Italy, performing robotic radical prostatectomies, partial nephrectomies cystectomies robotic and robotic packaging with orthotopic ileal neobladder, becoming reference of Tuscany robotic urologic surgery. An important role is reserved for the robotic surgery in the treatment of mediastinal diseases and Myasthenia gravis, often associated with thymic hyperplasia, achieving almost identical to those obtained with a traditional open-air approcchio percentage of complete remission of the disease involving the longitudinal sternotomy median.

The first surgical robot called da Vinci, in honor of Leonardo da Vinci, was developed in Silicon Valley by Intuitive Surgical and in 2000 he obtained the authorization of the American Food and Drug Administration (FDA) for use in laparoscopic surgery. The da Vinci Surgical System (sic) is a robotic surgical system made by the American company Intuitive Surgical. Approved by the Food and Drug Administration (FDA) in 2000, it is designed to facilitate complex surgery using a minimally invasive approach and is controlled by a surgeon from a console (Fig. 2a and 2b), (Da Vinci Surgical System, From Wikipedia).
The system is commonly used for prostatectomies and increasingly for cardiac valve repair and gynecologic surgical procedures. According to the manufacturer, the da Vinci System is called "da Vinci" in part because Leonardo da Vinci's "study of human anatomy eventually led to the design of the first known robot in history", (Akubue, 2011).

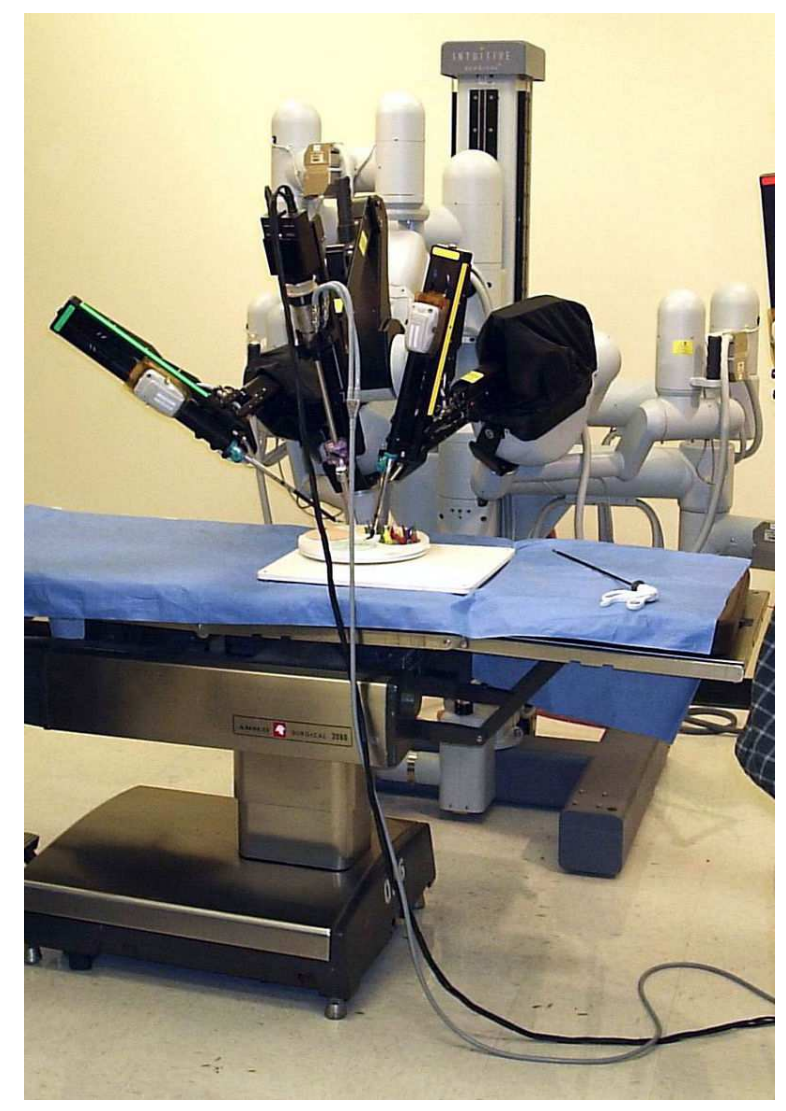

Fig. 1. Robotic surgery

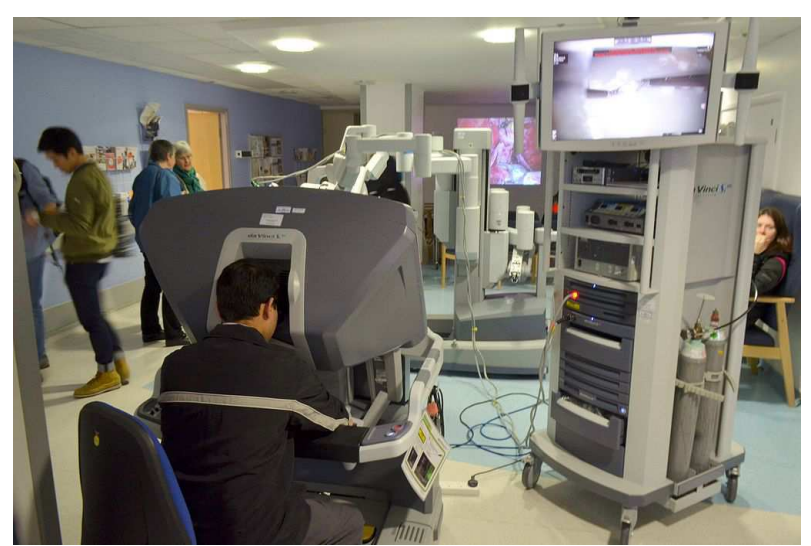

Fig. 2. (FDA) for use in laparoscopic surgery (a) Da Vinci patient-side component (left) and surgeon console (right) (b) a surgeon console at the treatment centre of Addenbrooke's Hospital 
The da Vinci Surgical System (Fig. 2a and 2b) of Intuitive Surgical is a robotic surgery system. It is used most commonly in the removal procedure of the prostate, heart valve replacement and in gynecological surgery procedures, but can also be used for any abdominal or thoracic surgical procedure. It consists of four robotic arms. Three of them are tools that keep objects such as scalpels, scissors, or Bovie electrocautery instruments. The fourth arm carries a camera with two lenses that allows the surgeon a complete stereoscopic vision from the console. The surgeon is sitting at a control panel and look through the crosshairs threedimensional image of the procedure while maneuvering the arms with two pedals and two hand controls.

The da Vinci Surgical System is normally used in the removal of the prostate, heart valve replacement and in gynecological surgery procedures. The da Vinci is an advantage in procedures that focus on a specific area of the abdomen or chest. The interventions that are not localized and which require extensive surgeon's mobility to the different areas are particularly disadvantageous in consideration of the time required for the preparation of the apertures Da Vinci. Surgeons are also experimenting with the da Vinci for the removal of the tumor in the liver and pancreas in the light of the sensitivity of the procedure, the number of blood vessels that the surgeon has to handle and the topical location of the procedure.

It promises that the da Vinci can be used in the future to effect real robotic surgery operations remotely. The possibility of remote operations depends on the availability to the patient of an apparatus da Vinci and someone able to practice the openings, but from a technical point of view the device would allow, for example, to a physician in the United States to operate on a patient in Antarctica.

The advantages of robotic surgery with the da Vinci system are (AB Medica):

- Lower margins post-surgical positive (measure indicating residual tumor cells at risk of tumor recurrence)

- Reduced hospital stay

- Less postoperative pain

- Lower risk of infection

- Less blood loss and fewer transfusions

- Smaller scars

- Better functional recovery of continence and erectile ability in prostate surgery

The da Vinci system by placing the device shared between several hospitals with a surgical transfer of know-how, can reduce the cost of Robotic Assisted Radical Prostatectomy (RARP), without compromising surgical results, even at the beginning of the learning curve.
Critics of robotic surgery argue that it is difficult to learn the use of the users and there are no studies showing that they are more effective than traditional laparoscopic surgery. The da Vinci system uses proprietary software, which cannot be changed, which limits the freedom of doctors to change the path of the operation In addition, its high cost (about \$ 2 million in the years 2010), makes it prohibitive for many institutions.

The manufacturer, Intuitive Surgical, has been criticized for having achieved a rapid approval by the FDA with a simplified procedure known as "premarket notification" (prior notification to commercialization) that is based on the assumption that the proposed product would be similar to products already previously approved. Intuitive was also accused of providing inadequate training and to encourage health facilities to reduce the number of supervised procedures before a doctor is allowed to use the system without supervision. It is also argued that some patients would have suffered damage due to accidental currents unleashed by inappropriate components of the surgical ends used by the system. Intuitive ribattutto that has the same type of undesirable current can also be originated by non-robotic laparoscopic procedures. A study published in JAMA-Journal of the American Medical Association claims that the side effects and blood loss in robotic hysterectomies are no better than those obtained by the traditional surgery, despite the much higher cost of the system. In 2013, the FDA began to investigate any issues related to the use of the da Vinci robot, including cases of death during surgery in which the device has been used; are pendants, also, a number of related legal cases to such events.

\section{Milan}

Robotic surgery is a real revolution, to which one can't do without, or a chimera by high costs for the National Health Service? This discussed the major Italian experts, meeting in Forlì for a convention during which they assessed the effectiveness of the technique that the expenditure required to buy it and keep it. "The conclusion to which we came-says Domenico Garcea, director of the Surgery Department AUSL Forlì and organizer of the meeting-is that the robot is a definite advantage, for which you pay a substantial figure. Several studies and Italian experiences have shown, however, that costs you can break down if it is a multi-disciplinary use, with a targeted selection of patients to operate and proper training of surgeons and all medical personnel." The price of the last model is around two million and 400 thousand euro and today there are about 50 hospitals, most of them public, who bought a robot. Some properties, such as the European Institute of Oncology and the San Raffaele in Milan, after careful medical and economic assessments have decided to buy a second. The hospital of Grosseto, then, has already taken the third. 


\section{The Pros and Cons of the Robot I}

"The benefits for patients-continues Garcea-are the same that can be achieved with the minimally invasive surgery: Smaller scars, fewer complications, shorter hospital stays, improved recovery times. The fact is that the robot gives the surgeon the ability to move, to turn a hand or an arm, $360^{\circ} \mathrm{C}$ : Something humanly impossible for a human being. Or to arrive at a precise point without doing contortions strenuous, take tough positions to hold for hours. It also gives us a three-dimensional vision, with a visual and a perfect light. unfavorable aspects for the sick, compared to traditional surgery, there are none. "The cons, however, exist for the hospital's coffers or the NHS: To date, when it comes to robotic surgery we are a monopoly. The only available specimen is the Da Vinci, it arrived on the market at the end of the nineties and then updated in five models. As long as the patent expires there is no competition, even if "now, the robot purchase is almost never be borne by the health authorities, but it is often the result of a donation by private entities-comments Andrea Coratti, director of general surgery hospital of Grosseto Misericordia and pioneer, along with Pier Cristoforo Giulianotti, the Italian robotic surgery. They are mainly operating costs that should be cut." (AB medica).

\section{Possible Solutions to Reduce Costs}

The robot therefore, at least for now, involves too high costs because many hospitals can afford to buy one? Not according Franca Melfi, an expert in thoracic robotic surgery, which contains data a study sponsored by the Thoracic Surgery, University of Pisa: "If you use a machine for a number of applications per year, identified in about 300, surgery robotics is not only expensive, but saves resources-explains. Based on this principle, the Tuscany Region has approved a project to expand the robotics "economically compatible." In practice, the Region has allocated funds for the purchase of new robots, which will be used, however, in the interdepartmental way: Physically placed in some facilities will be used in turn by most surgeons to more hospitals. "The principle, therefore, is to depreciate the investment using the Da Vinci for a large number of applications every year, for different diseases and in patients specially selected." These devices today can and must have a variety of applications-precise Giulianotti, started from Grosseto and came three years ago in Chicago, where he directs the Department of General Surgery and robotics at the University. For example in urology, gynecology, cardio-thoracic and gastrointestinal surgery, pediatrics or oncology. In the coming years, then, the robot can always use more in the cervical region, thyroid, in otorhinolaryngology and the major operations of the colon, liver, pancreas and kidneys."

\section{Reserved for 'Formula one Drivers'}

The conclusions of the conference emerges a final rule: To use the robot serves a clear and sufficient training. The greater the specialization of the surgeon, the better is the way to use the equipment. "It's a little 'how to drive a car in a race of Formula One: We are not all capable and, in any case, to be good pilots must prepare a lot and constantly updated" exemplifies Garcea. "You have to buy dexterity and, as in any field, one learns and improves with practice-explains Paolo Bianchi, Unit Director of the European Institute of Oncology minimally invasive surgery of Milan and founding member of the Clinical robotic surgical association. In this way not only we ensure the best outcome for patients, but-once againrestrict the costs (of wear, expensive disposable equipment related apparatus, time). The robot, in conclusion, is a tool that essentially facilitates the surgeon and makes it possible to perform minimally invasive treatments that more and more complex, with traditional laparoscopic technique, only a few surgeons would be very long and practicable many experts", (Chirurgia robotica, la rivoluzione in medicina).

Very few thousand worldwide, fifty in Italy. It is of very high precision robotic equipment and technology that assist surgeons in the most complex and important treatments.

Integrated systems of video-shooting, laser, mechanical arts help the surgeon to realize interventions otherwise almost impossible, such as the removal of tumors defined until a short time ago inoperable, or by the very high cost, both in economic terms-think the cost of a prolonged hospital stay by an invasive intervention-both as endurance of physical pain for patients (Chirurgia robotica, la fantascienza incontra la medicina).

Of all these tools we talk during the VII European Congress of laparoscopic and robotic surgery going on these days in Rome. More than 400 doctors have come from all over the world, who will attend to some of the most complex operations.

You can ask a question to the forum specialists or complexes made at the clinic Pius XI in Rome, explains Vito Pansadoro, surgeon urologist and president of Congress, "is the first time ever that an international meeting is designed to bring the laparoscopy and robotics to its extremes. Are all cases the operability limits, that only thanks to surgeons of the highest caliber have become possible now $60 \%$ of worldwide operations performed with the Da Vinci robot-explains Pansadororelate to prostate cancer, in the United States are 1 $86 \%$, followed by the removal of kidney tumors, cystectomies and stenosis of the urinary tract".

Highs are the costs of a surgeon robot, about two million dollars, in addition to $\$ 150,000$ a year for maintenance. However, in some cases it is indispensable: "We have solved cases with a narrowing of the urinary 
tract due to endometriosis or patients operated without success already four or five times," says Prof. yet. Pansadoro. There are many efforts in areas of the body difficult to reach; such as replacing orthotopic bladder, lymph node dissection and tumors of the testis, the partial or total nephrectomy, "in which laparoscopy becomes necessary when we have only one kidney," recalls Pansadoro. With regard to kidney surgery, the professor adds: "Today $60-70 \%$ of kidney tumors are discovered by chance through an ultrasound or a CT performed for other purposes and the possibility of intervening with ever less invasive capacity represents the next surgery".

The latest invention in terms of robotics applied to medicine is the "fetus-robots." Designed by researchers University of Tokyo, he is able to simulate the movements of the fetus in the amniotic fluid. The goal is to study the child's coordination skills, treat early disease of the newborn and improve intrauterine and neonatal surgery. If Japanese MPRE is the robot-nurse prototype, a humanoid upper $155 \mathrm{~cm}$ and $70 \mathrm{~kg}$ of weight. The automaton is able to collect, move and transport small or large objects, up to a weight of 30 kilograms. The robot may soon be used in hospital wards for the transport of materials, drugs and patients.

Finally, the dream to travel inside the human body has already become reality. Israeli scientists have invented a pill equipped with a miniature camera, once swallowed, travels and films him in the gut, avoiding the introduction of probes for diagnostic purposes such as gastro-scopy and colonoscopy (MEDICINA ROBOTICA).

\section{Materials and Methods}

\section{Robotics Urology and Surgery}

The most important national and international guidelines, especially in the field of urology and surgery, indicate rectal examination as a decisive diagnostic maneuver and always be done when there is a pain especially at the lower abdominal wall (Seehusen, 2007), in the persistent conditions of lower urinary tract (Zanollo et al., 1991), in anorectal disorders (Beretta, 2014) and, even in the absence of symptoms, however, it should be part of the urologic evaluation especially in the diagnosis of prostate cancer and in general surgery of colorectal cancer (Nickel, 2010) (Fig. 3), (DRE: Its importance in the disciplines uro-andrological).

Andro-urology field, but also in general surgery, a precise and targeted clinical examination allows in many cases to suspect and even diagnose cancerous lesions indicate other diagnostic tests are able to confirm or refine the suspected diagnosis of the clinical problem addressed, the figure' urgency of the problem that we must address, who is a specialist on which eventually orient the patient: Urologist, urologist, proctologist, gastroenterologist, surgeon and finally, for example in the presence of significant psychological or psychiatric problems, to assess whether it should call It also considers these professionals.

It 'important for the urologist and andrologist not underestimate the clinical maneuver that must always be preceded by a careful and precise examination of the abdomen with the patient lying in a supine position; in this way you will be able to assess important clinical signs (sovradistensioni bladder, timpanismi, painful points, scars, hernias, inguinal lymphadenopathy) and examine the genitals of the male (penile structure, aspect of the glans, the foreskin, the external urethral meatus, the scrotum, testes characteristics, the epididymis, the cord and more). After this phase, the digital rectal examination and then is invited the patient to fold and to spread the knees in order to expose as much as possible to the perineum. Several clinical indications spasticity of the lower limbs, obesity, neurological injury, such as a spinal paralysis or paraplegia or ankylosis hip joint, can prevent such maneuvers and make it difficult to establish regular and normal digital rectal examination.

In these cases it may be necessary to use other more particular positions as the knee-chest position from supine or lateral position (generally indicated position in the field Proctological), (Reilly, 2003).

First major DRE is also to evaluate well the perineal area in this way are seen any lesions of the skin infections, secretions, fistulae, abscesses, various ulcers, warts, psoriasis lesions or traumatic, hemorrhoids, fissures or marische.

Assessing the presence of pain, let us always remember that the sensitivity of the perineum are neurologically linked directly to mielomeri sacral.

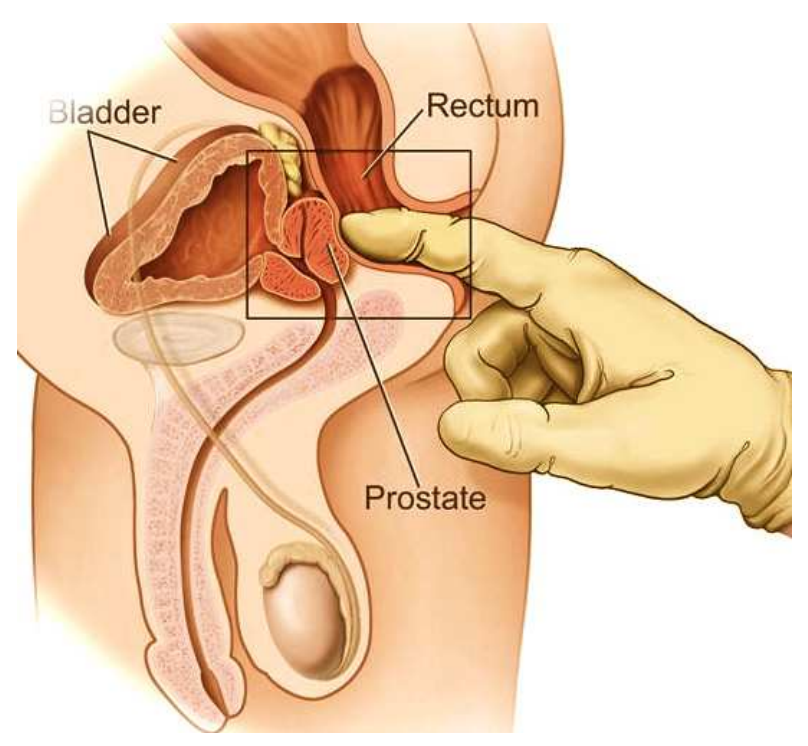

Fig. 3. DRE: Its importance in the disciplines uro-andrological 
Therefore the inspecting digital rectal ampoule, only in this way you will have the information more specific and targeted on the anal sphincter, the contents of the rectal ampulla, the characteristics of the walls of the rectum, the prostate lateral lobes, vesicle seminal and, sometimes, even the ureters.

\section{The Anal Sphincter}

You can only evaluate the external striated muscles in its component, a careful assessment of this anatomical region can show the sacral nerve reflex arc integrity and indirectly also give us some information on the striated sphincter of the prostatic urethra.

Are considered muscle tone at rest, the beanza and anal rhyming skills, voluntary contraction of the muscle when you start browsing, the voluntary contraction exploring real and reflex contraction.

They are valued as follows:

The superficial anal reflex: It introduces, in a swift and sudden, a finger to a few millimeters, in the anus and are practiced with this of small strokes in the sphincter level and, by doing so it has a reflex contraction that should generally expresses an integrity mielomeri S4-S5.

The reflection bulb-cavernous or Deep anal sphincter; in this case the explorer finger is in the anal canal while with the other hand you make small tweaks to the level of the glans, the tip of the penis; in this case it is triggered a reflex contraction of the anus indicating a neurological integrity of mielomeri S2-S3-S4.

Other evaluated reflex is triggered by the stretching (forced stretching) bilateral or unilateral skin located at the perineal level (between the anus and the scrotum) that triggers a massive contraction of the pelvic floor muscles with a final movement due to prevailing activity the levator ani that door up around the perineum. Then the request to increase the intra-abdominal pressure with classical Valsalva maneuver will tell if the pelvic support of the central perineum, always linked to the pelvic diaphragm muscles, it presents a supporting deficits for example to the patient's chronic habit of exercising strong intra-abdominal pressure in the presence of chronic constipation or neurological problems that indicate a denervation of the pelvic floor and accordingly a secondary overall muscle flaccidity (Fig. 4).

The pain, when doing a rectal examination, may be due to the procedure itself, which is still somewhat invasive, but we must never forget the combination of possible psychological problems, related to the particular sense of decency or some patient's prejudices, to a particular and lively reflex muscle contraction, to the fact that it is not adequately lubricated the finger which explores, for any unexpected stretching of some body hairs, in the presence of hemorrhoids and rectal inflammation, yet finally consider the presence of a stenosis, that is, a Refinement of the anal canal.

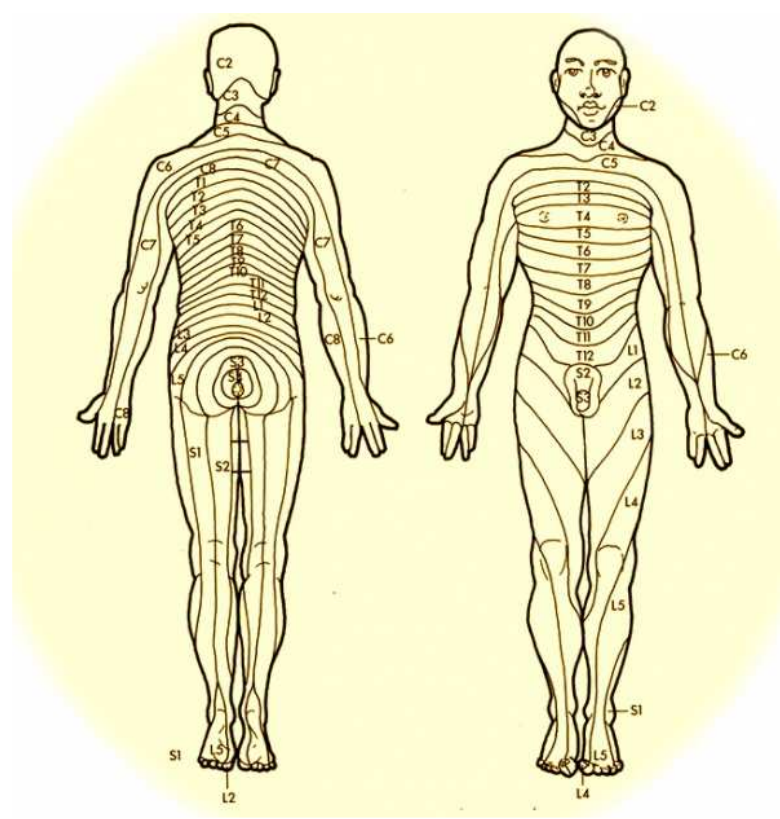

Fig. 4. The anal sphincter

Evaluation of the content present in the bulb rectal.

Generally, if the patient does not feel the urge to have a bowel movement, rectal ampoule is empty.

If there are feces they must be well-considered and should be given their characteristics.

Often, especially the elderly person in acute retention of urine also presents a situation of clogging and over-distension of the rectum, this situation often alone triggers a reflex, through a peripheral neurological mechanism, a paresis of the bladder muscle, the detrusor and consequently also the acute retention of urine in a person who has perhaps incomplete chronic retention of urine.

Over DRE always assess the presence of blood and the color of the fecal material that possibly there is (Chiu et al., 2015).

\section{Examination of the Ampoule Rectal Wall}

As first thing is to be evaluated the state of the mucosa, its "smoothness" on the underlying levels, the presence of strictures, stenoses, areas of increased consistency, fixed masses, pain in some particular points of the wall, their inflammatory areas, such as abscesses pararectal and other.

Without these general maneuvers are then used an assessment more properly uro-andrology.

The exploration of the prostate.

The prostate is easily perceived through the wall of the rectal ampulla, it is usually divided into two side lobes by a median groove and has an elastic hard consistency while its surface is smooth and uniform and is generally perceived to approximately $3-4 \mathrm{~cm}$ from the 
anus; if overweight patient may sometimes have problems to explore it; its palpation, in normal situations, usually does not trigger pain, however we have to remember now that the trans-rectal examination means you can control the rear wall of the side lobes.

So we can verify in general the shape reminiscent of a chestnut with the apex towards the anus.

Difficult to appreciate the middle lobe; through the difficult DRE also quantify the weight that generally in the adult male is $15-20$ grams.

Usually for its volume it is given relating it to chestnuts, plums, oranges and tangerines or described with simple factors of increase for two for three, etc.

The characteristics of the gland surface may already be a sign of certain diseases, for example irregular nodules can indicate different problems ranging from cancer to inflammation acute or chronic; still fine granules and hard suggest various calculations or fibrosis. Another fact to consider is the size of the gland if it lasts and wooden you can suspect a tumor, whether fibrous chronic prostatitis or a sclerosing adenoma; if soft areas and "flabby" you think to an acute prostatitis.

In a normal prostate limits are well defined and precise but become inaccurate and blurred in the presence of inflammation and cancer, when the cancer is very advanced you lose all the limits and you have a "slab" hard, hard and it lacks the physiological flow with the underlying and surrounding floors.
The pain may be present in case of inflammation, if alive and burning suggests an acute prostatitis or intraprostatic abscesses. The pain however is generally not present when we benign prostatic hypertrophy, but may be present so overwhelming and not well defined when there are tumors and even when there are numerous calcifications intraprostatiche (Walsh, 2014).

With rectal examination in some rare special situations you can also hear the seminal vesicles, which are located at the rear and the top edge of the prostate on both right and left.

If there is an inflammation, a cyst or a tumor of the vesicles (very rare) it is unable to locate them better than the prostate gland.

Finally towards the side edge and top of the prostate on both sides, in particular pathological situations, you can palpate the pelvic ureters in their terminal portion.

This happens in the presence of fallen stones up to pre-bladder level, when you have a megaureter that marked dilatation dell'uretre terminal (Fig. 5).

As a last diagnostic since there can be given by the evaluation of a rectal examination, finally we have to remember an eventual secretion, which may leak from the urethra after palpated the prostatic region, for example a transparent secretion and stringy, also called prostatorrea, or we can observe a purulent secretions, blood or still can be discharged prostatic secretions and then you also talk of spermatorrhea (Little et al., 2004).

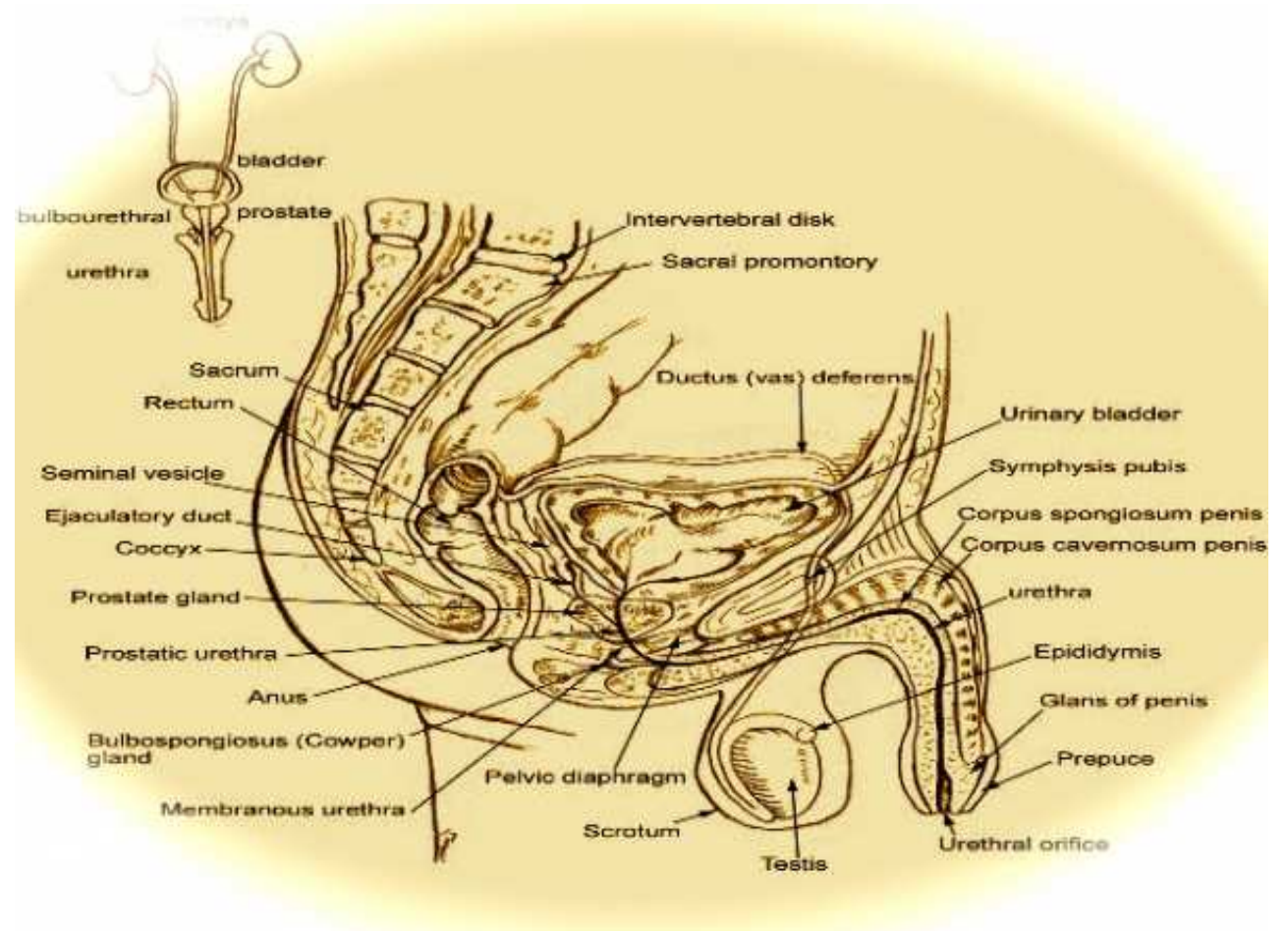

Fig. 5. The exploration of the prostate 


\section{Robotics Surgery Mechanical System of Parallel Structeres}

Moving mechanical systems parallel structures are solid, fast and accurate. Between parallel systems it is to be noticed Stewart platforms, as the oldest systems, fast, solid and precise. The work outlines some few main elements of Stewart platforms. It begins with the geometry platform and structure and then one presents the kinematic elements of it, positions, velocities and accelerations. If a structural motto element consists of two moving elements which translate relative, drive train and especially dynamic it is more convenient to represent the motto element as a single moving component. One has thus seven moving parts (the six motto elements or feet to which is added mobile platform) and one fixed. The whole work is based on knowledge of analytic geometry, so we can consider this work as a study of the applied mathematics.

Figure 6 shows the kinematic diagram of a mobile mechanical parallel system, heving all 12 kinematic couplings (which connect the six motor legs with the two platforms fixed and mobile) type ball joints (couplers sphere to sphere allowing all rotations possible and do not give permission to produce any translation), practically couplers third grade $(\mathrm{C} 3)$. Kinematic engines couplings (six in number) may be constructed in two versions: $\mathrm{C} 5$ or C4 (Petrescu and Petrescu, 2011; 2012; 2013).

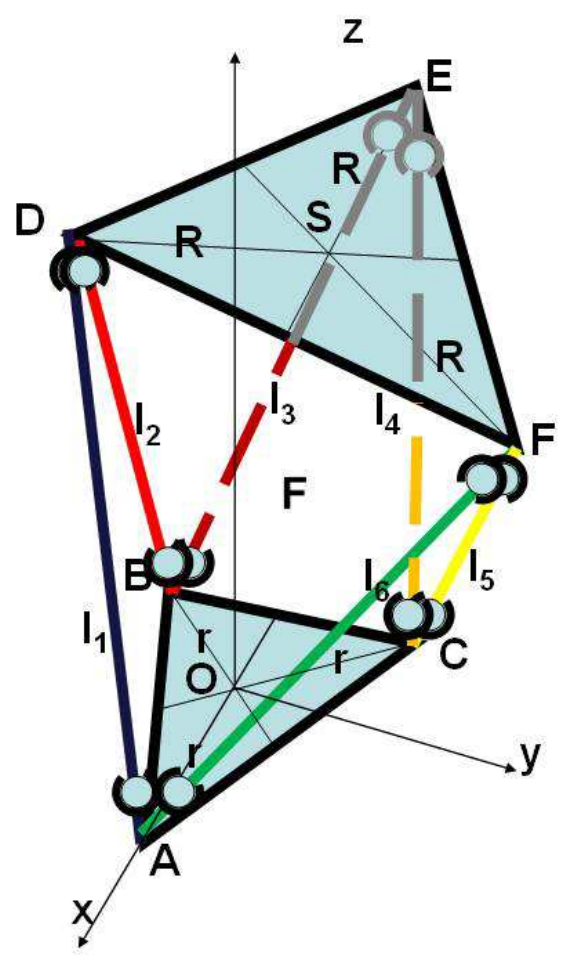

Fig. 6. Kinematic diagram of a mobile mechanical parallel system
Couplings sphere in sphere (ball joints) allow rotations in space on all three axes and stop all translations. They are more difficult to achieve in terms of technology, are more expensive and generally have shorter life, their wear being quite fast (even if the contact surface of the sphere is large sphere type). But have the great advantage of a reduced size (mass and low volume) (Fig. 7). Their life can be extended by optimal design, by careful processing, through a suitable lubricant, or using special materials (Petrescu et al., 2009).

For a parallel system 12 ball joint (C3) and 6 motor couplings (C5) only the translation the grade fifth, the mobility of the system (spatial mechanism) is given by the general formula (1), (for a spatial mechanism family 0):

$\left\{\begin{array}{l}M_{0} \\ =6 \cdot m-5 \cdot C_{5}-4 \cdot C_{4}-3 \cdot C_{3}-2 \cdot C_{2}-1 \cdot C_{1} \\ =6 \cdot m-5 \cdot C_{5}-3 \cdot C_{3}=6 \cdot 13-5 \cdot 6-3 \cdot 12 \\ =78-30-36=12\end{array}\right.$

Where $\mathrm{m}$ represents the number of the moving parts of the mechanism (system), in this case where $\mathrm{m}$ is equal to 13 , because the six legs each consisting of two elements (i.e., $6 * 2=12$ ) and one of the two platforms (the upper) is also mobile (representing the thirteenth movable element of the system). Of the $12^{\circ} \mathrm{C}$ of freedom of the system only 6 are active (they representing the linear movements of the linear motors). The other six degrees of freedom are passive (does not indicate the need to use additional actuators to achieve them). They are basically evidenced by six additional rotational movements of the six legs, each leg consists of two elements cinematic considered as a solid, may rotate freely between its two ball joints (by which is connected to the two platforms: Based on the fixed and movable above), (to follow Fig. 8). These additional rotational movements allow the kinematic movements of the system.

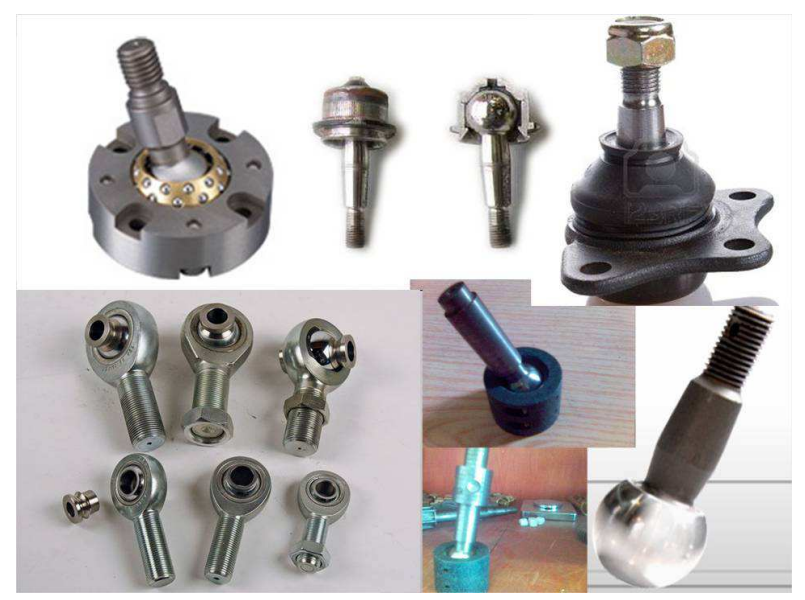

Fig. 7. Couplings sphere in sphere (ball joints) allow rotations in space on all three axes and stop all translations 
Basically, it is used instead of couplers translation engines (C5) some motor couplers type cylindrical (C4) which in addition to translational movement, allow and a relative rotation between the two bars of the motor coupling. Linear actuators are constructed so that each allows a relative rotation between its two active bars. Motor movement is the linear, but is allowed and a relative rotation in motto element. In this situation will disappear the six couplers of grade V (C5), being replaced entirely with swivels cylindrical grade IV (4), (Fig. 9). The formula of degree of mobility takes the appearance (2):

$$
\left\{\begin{array}{l}
M_{0} \\
=6 \cdot m-5 \cdot C_{5}-4 \cdot C_{4}-3 \cdot C_{3}-2 \cdot C_{2}-1 \cdot C_{1} \\
=6 \cdot m-4 \cdot C_{4}-3 \cdot C_{3}=6 \cdot 13-4 \cdot 6-3 \cdot 12 \\
=78-24-36=18
\end{array}\right.
$$

Mechanism enhances its mobility, but only six of these degree of mobility are active (they refer to linear movements imposed by the six actuators). In this case the mechanism has 12 passive rotational movements (12 passive degree of mobility).

Both variants presented are functional and have a high dynamic. Now is frequently used a combined system more rigid (in terms of dynamic) and economic, in which six of the ball joints (C3) are replaced with six universal joints, that are couplings of the Class IV (C4). So of the 12 ball joints $\mathrm{C} 3$ remain now for the new system to use half (six ball joint, couplers C3) and the other six will be now of the class IV (type universal joints) and together with the old cylindrical joints with motors (C4) will achieve now to the Stewart platform 12 couplers type $\mathrm{C} 4$. Mobility will be given now by formula (3), Garcia et al., 2013):

$$
\left\{\begin{array}{l}
M_{0}=6 \cdot m-5 \cdot C_{5}-4 \cdot C_{4}-3 \cdot C_{3}-2 \cdot C_{2}-1 \cdot C_{1} \\
=6 \cdot m-4 \cdot C_{4}-3 \cdot C_{3}=6 \cdot 13-4 \cdot 12-3 \cdot 6 \\
=78-48-18=12
\end{array}\right.
$$

The vast majority of parallel platforms type Stewart has today 12 universal joints and 6 cylinder motor couplings, all of them being kinematic couplings of the grade IV (C4). Disappearing joints C3 and couplings C5 engines and remain only universal joints and couplings cylinder engines, all of the kinematic class C4 (Fig. 10).

Universal joints can be used constructively in several ways (Fig. 11).

The formula for calculating the mobility is writing now greatly simplified by expression (4):

$$
\left\{\begin{array}{l}
M_{0} \\
=6 \cdot m-5 \cdot C_{5}-4 \cdot C_{4}-3 \cdot C_{3}-2 \cdot C_{2}-1 \cdot C_{1} \\
=6 \cdot m-4 \cdot C_{4}=6 \cdot 13-4 \cdot 18 \\
=78-72=6
\end{array}\right.
$$

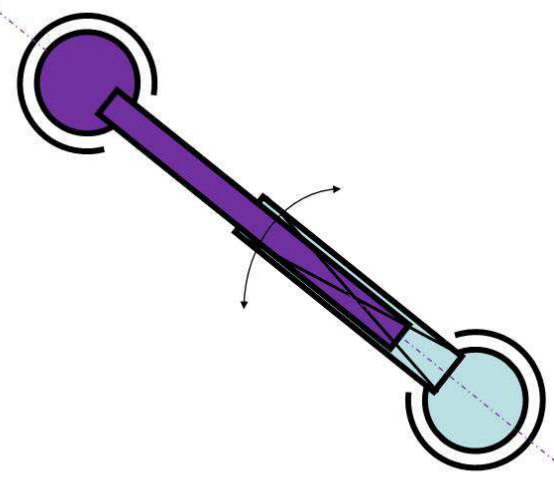

Fig. 8. Additional rotational movement of a leg between the two ball joints

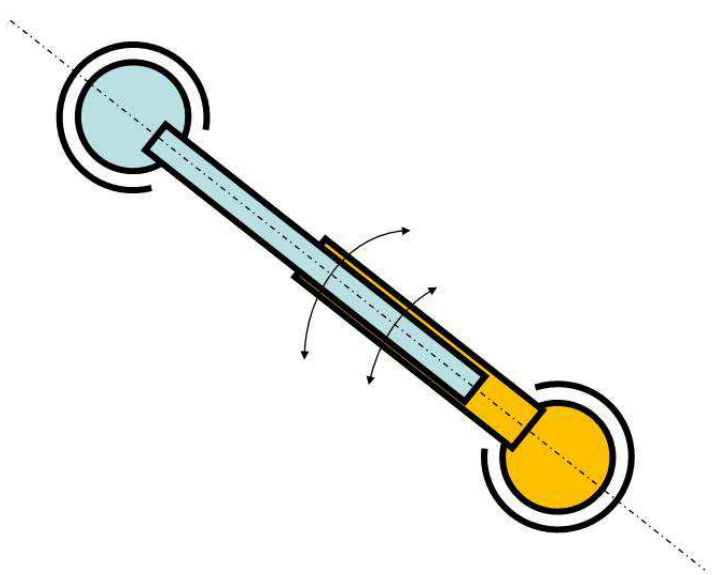

Fig. 9. Two additional rotational movements of a leg: first one is the leg rotation between the two ball joints; and the second one is the relative rotation between the two elements (cylindrical bars) of the leg

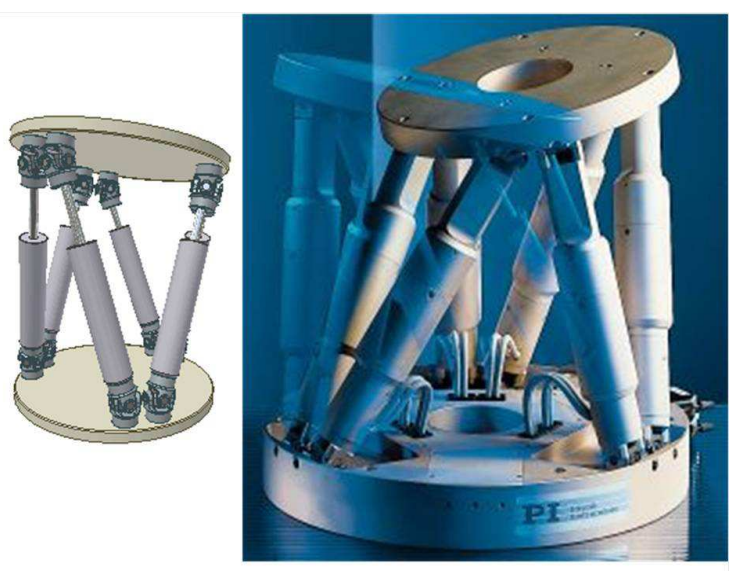

Fig. 10. Modern platform Stewart which uses only (18) couplings grade $4(\mathrm{C} 4)$ 


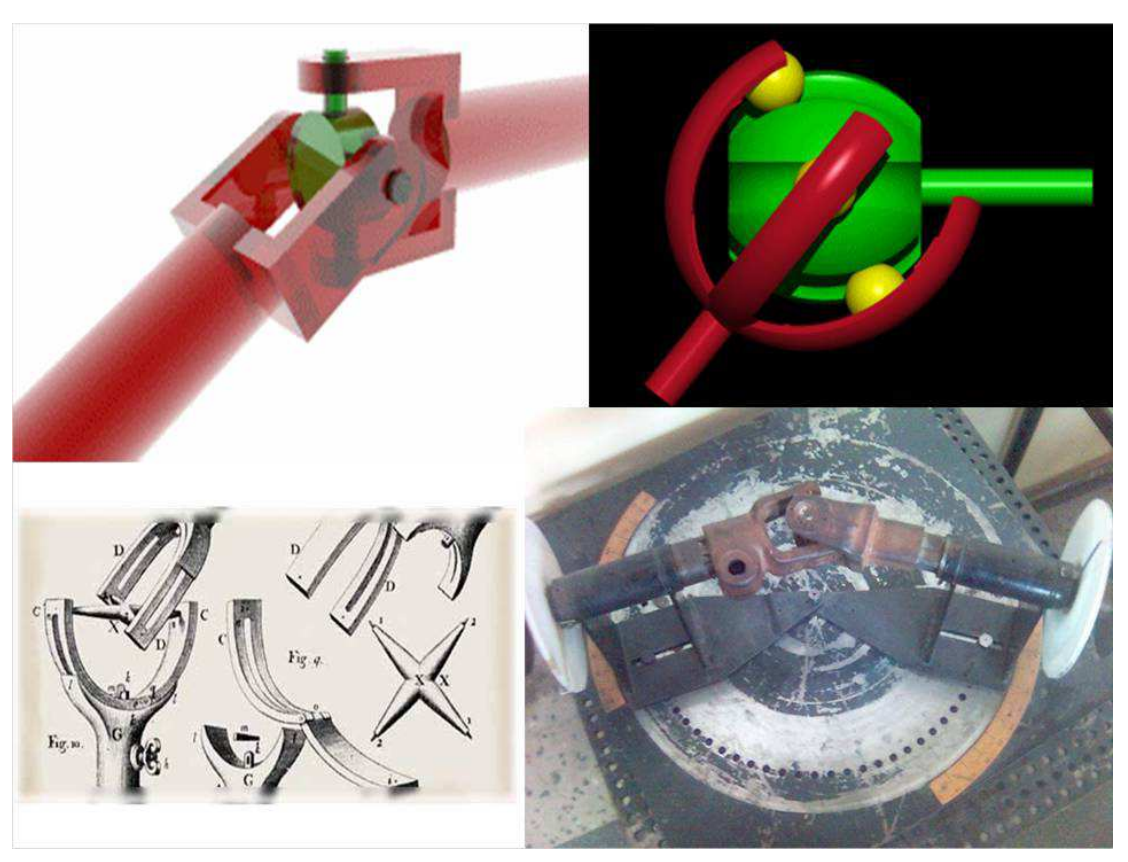

Fig. 11. Universal joints (their constructive diversity is high)

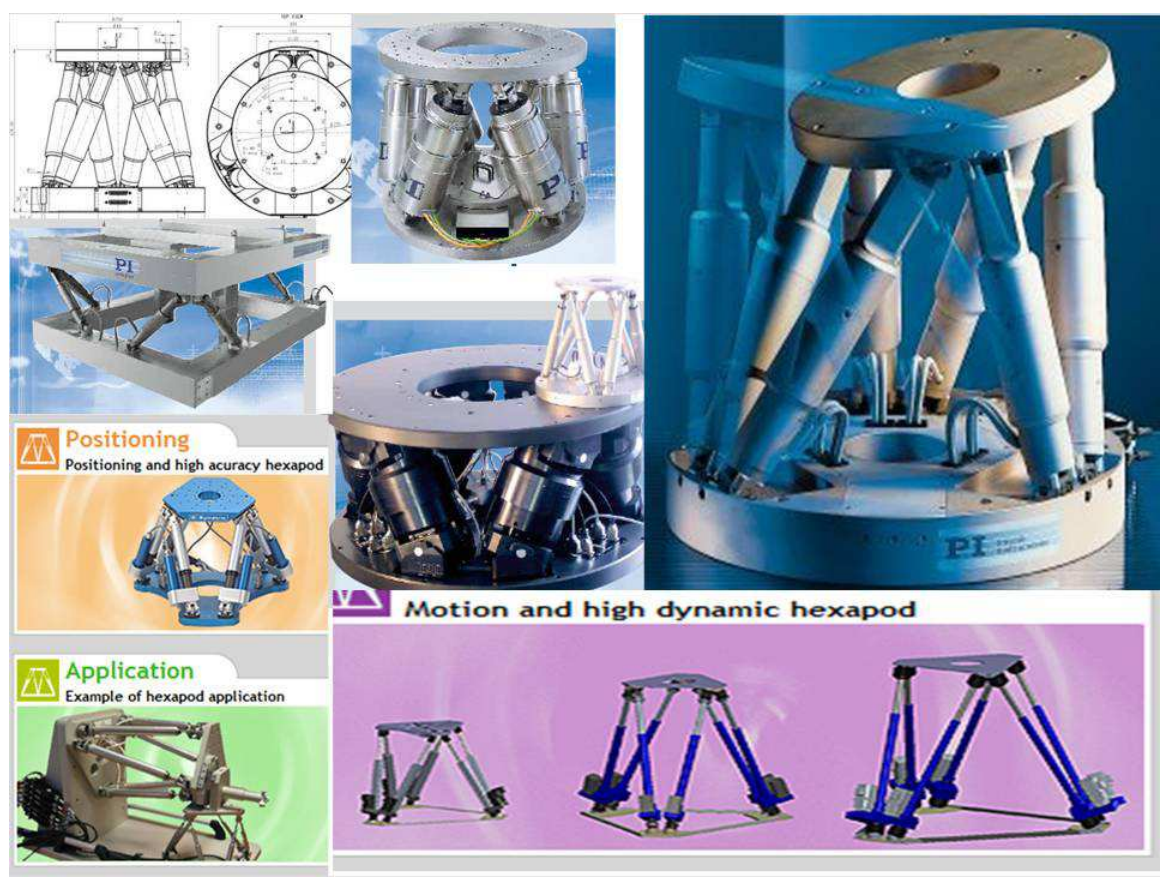

Fig. 12. Various platforms type Stewart 12).

Today, they use various systems of type Stewart (Fig.

All these platforms achieve high speeds in work with a good dynamics. Their balancing is simpler and higher. Systems are more robust and more accurate. In Fig. 13 one can see a Stewart platform with nine legs.
Its structural formula is given by relation 5 :

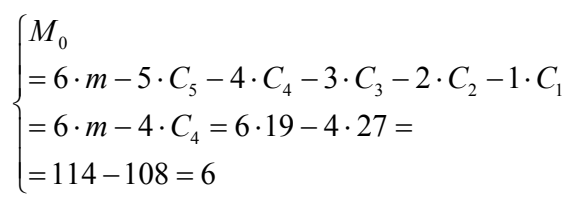


From the structural formula can be seen that only six legs are engines and the other three are driven by the mobile platform element (their role being more to increase system stability). Or all legs are motors and their movements must be well coordinated and synchronized with other main actuators (Calculation of movement in this system is much more difficult). To have the mobility of this mechanism 9 instead of 6 as three universal joints must be replaced with three spherical kinematic couplings (see relation 6):

$$
\left\{\begin{array}{l}
M_{0} \\
=6 \cdot m-5 \cdot C_{5}-4 \cdot C_{4}-3 \cdot C_{3}-2 \cdot C_{2}-1 \cdot C_{1} \\
=6 \cdot m-4 \cdot C_{4}-3 \cdot C_{3}=6 \cdot 19-4 \cdot 24-3 \cdot 3 \\
=114-96-9=9
\end{array}\right.
$$

Stewart systems can keep their balance during rapid movements loaded with a heavy load (Fig. 14).

\section{Inverse Kinematics}

\section{Determining Positions and Displacements}

In Fig. 15 one can see a simple theoretical model.

In Fig. 16 is determined the position parameters (spatial Cartesian coordinates) for fixed points A, B, C. For point $\mathrm{A}$ is obtained $x=r$ and $y=z=0$. For point $\mathrm{B}$ using relations (7) and to determine the coordinates of $\mathrm{C}$ are considered system (8) (Wang et al., 2013):

$$
\begin{aligned}
& \left\{\begin{array}{l}
x_{B}=-\frac{1}{2} \cdot r \\
y_{B}=-\frac{\sqrt{3}}{2} \cdot r \\
z_{B}=0
\end{array}\right. \\
& \left\{\begin{array}{l}
x_{C}=-r \cdot \sin 30^{\circ}=-\frac{1}{2} \cdot r \\
y_{C}=r \cdot \cos 30^{\circ}=\frac{\sqrt{3}}{2} \cdot r \\
z_{C}=0
\end{array}\right.
\end{aligned}
$$

For mobile platform DEF (Fig. 17) can write Equation 9. Basically were written distances between the peaks of the triangle DEF Cartesian coordinate space:

$$
\left\{\begin{array}{l}
\left(x_{D}-x_{F}\right)^{2}+\left(y_{D}-y_{F}\right)^{2}+\left(z_{D}-z_{F}\right)^{2}=3 \cdot R^{2} \\
\left(x_{D}-x_{E}\right)^{2}+\left(y_{D}-y_{E}\right)^{2}+\left(z_{D}-z_{E}\right)^{2}=3 \cdot R^{2} \\
\left(x_{E}-x_{F}\right)^{2}+\left(y_{E}-y_{F}\right)^{2}+\left(z_{E}-z_{F}\right)^{2}=3 \cdot R^{2}
\end{array}\right.
$$

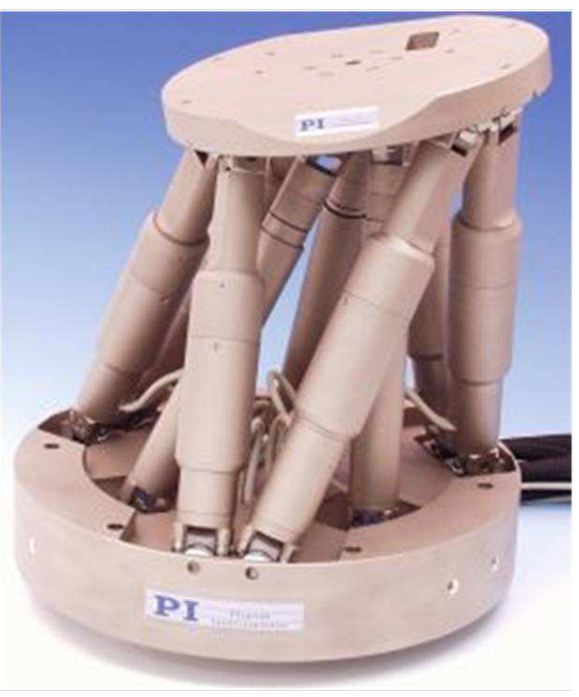

Fig. 13. A Stewart platform with nine legs

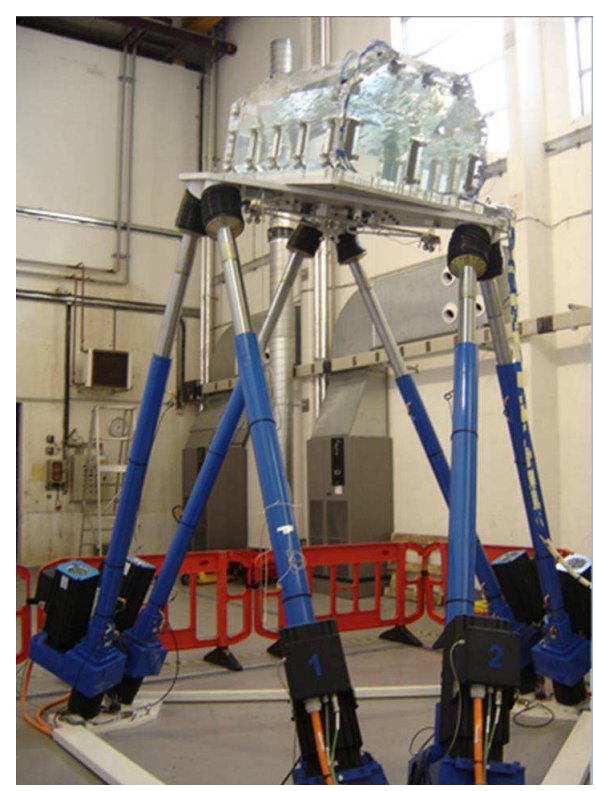

Fig. 14. A high loaded Stewart platform

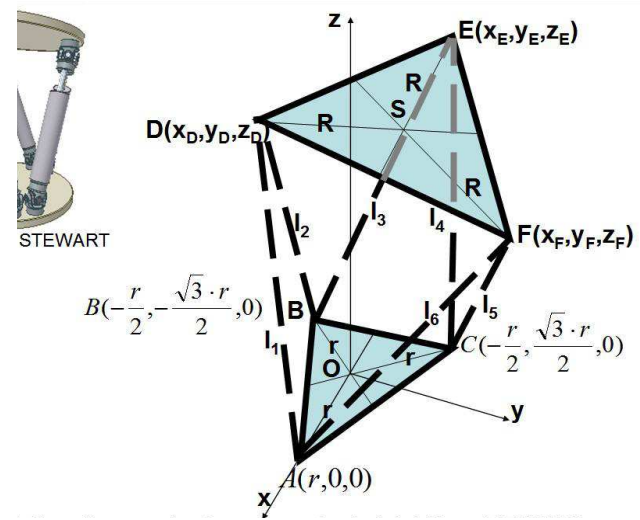

Fig. 15. A simple theoretical model 


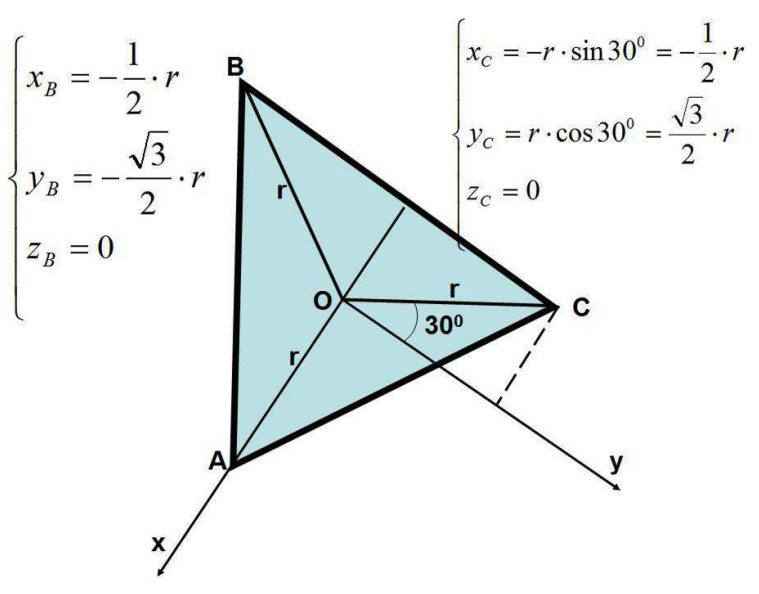

Fig. 16. Determining the fixed shelf

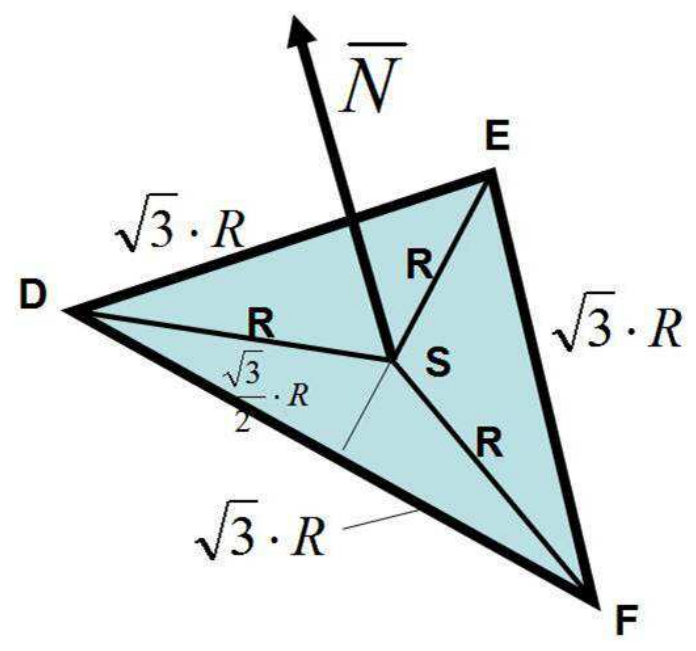

Fig. 17. Determining the mobile shelf

The process was repeated but this time there were writing distances between mobile triangle center $\mathrm{S}$ and each vertex of the triangle DEF (eq. system 10):

$$
\left\{\begin{array}{l}
\left(x_{D}-x_{S}\right)^{2}+\left(y_{D}-y_{S}\right)^{2}+\left(z_{D}-z_{S}\right)^{2}=R^{2} \\
\left(x_{E}-x_{S}\right)^{2}+\left(y_{E}-y_{S}\right)^{2}+\left(z_{E}-z_{S}\right)^{2}=R^{2} \\
\left(x_{F}-x_{S}\right)^{2}+\left(y_{F}-y_{S}\right)^{2}+\left(z_{F}-z_{S}\right)^{2}=R^{2}
\end{array}\right.
$$

Write now DEF plan equation under general form (11), where $\mathrm{D}$ is any point of the plan, $\mathrm{S}$ is a particular (central) of the plane and the vector $\mathrm{N}$ is the vector perpendicular to the plan, considered in specifically chosen point $\mathrm{S}$. The geometrical parameters (scalar) of position $(\alpha, \beta, \gamma)$ of the vector $N$ are known (Cao et al., 2013).

The general equation of a plan say that any right line of plan scalar multiplied by the vector $\mathrm{N}$ (perpendicular on the plan) generates the product 0 :

$\overline{D S} \cdot \bar{N}=0$
Will be assigned successive to the point $\mathrm{D}$ the values D, E, F and plan Equation 11 written scalar will receive forms (12):

$$
\left\{\begin{array}{l}
\left(x_{D}-x_{S}\right) \cdot \alpha+\left(y_{D}-y_{S}\right) \cdot \beta+\left(z_{D}-z_{S}\right) \cdot \gamma=0 \\
\left(x_{E}-x_{S}\right) \cdot \alpha+\left(y_{E}-y_{S}\right) \cdot \beta+\left(z_{E}-z_{S}\right) \cdot \gamma=0 \\
\left(x_{F}-x_{S}\right) \cdot \alpha+\left(y_{F}-y_{S}\right) \cdot \beta+\left(z_{F}-z_{S}\right) \cdot \gamma=0
\end{array}\right.
$$

Scalar parameters $x_{S}, y_{S}, z_{S}, \alpha, \beta, \gamma$ are known. With systems (12) and (10) can be determined immediately scalar parameters of a mobile point on the circle, choosing the initial determination of point $D$, for example. Must this point be known (positioned) at least through one of its coordinates. Suppose for example $Z_{D}$ coordinate known (known propensity of mobile plan by $\alpha, \beta, \gamma$, one know where the focus $\mathrm{S}$ must be, knowing $x_{S}, y_{S}, z_{S}$, but must be known and $z_{D}$ height of a point on the mobile circle). Determine then the other two scalar coordinates $x_{D}$ and $y_{D}$. One use the system (13) formed by the first relation of the system (12) and by the first equation of the system (10):

$\left\{\begin{array}{l}\left(x_{D}-x_{S}\right) \cdot \alpha+\left(y_{D}-y_{S}\right) \cdot \beta=\left(z_{S}-z_{D}\right) \cdot \gamma \\ \left(x_{D}-x_{S}\right)^{2}+\left(y_{D}-y_{S}\right)^{2}=R^{2}-\left(z_{D}-z_{S}\right)^{2}\end{array}\right.$

To solve (13) are introduced notations (14):

$$
\left\{\begin{array}{l}
x=x_{D}-x_{S} \\
y=y_{D}-y_{S} \\
\theta=\left(z_{S}-z_{D}\right) \cdot \gamma \\
L^{2}=R^{2}-\left(z_{D}-z_{S}\right)^{2}
\end{array}\right.
$$

From (13) with the annotations (14) obtains the system (15), which is solved sequentially by relations (16) which leads to an equation grade 2 with unknown $\mathrm{y}$, which solution is given by the first two relations of the system (17), while the third relation of the system (17) calculates $x$ :

$\left\{\begin{array}{l}\alpha \cdot x+\beta \cdot y=\theta \\ x^{2}+y^{2}=L^{2}\end{array}\right.$

$\left\{\begin{array}{l}x=\frac{\theta-\beta \cdot y}{\alpha} x^{2}=\frac{\theta^{2}+\beta^{2} \cdot y^{2}-2 \cdot \theta \cdot \beta \cdot y}{\alpha^{2}} \\ \theta^{2}+\beta^{2} \cdot y^{2}-2 \cdot \theta \cdot \beta \cdot y+\alpha^{2} \cdot y^{2}=\alpha^{2} \cdot L^{2} \\ \left(\alpha^{2}+\beta^{2}\right) \cdot y^{2}-2 \cdot \theta \cdot \beta \cdot y-\left(\alpha^{2} \cdot L^{2}-\theta^{2}\right)=0\end{array}\right.$

$\left\{\begin{array}{l}y_{1,2}=\frac{\theta \cdot \beta \pm \sqrt{\theta^{2} \cdot \beta^{2}+\left(\alpha^{2}+\beta^{2}\right) \cdot\left(\alpha^{2} \cdot L^{2}-\theta^{2}\right)}}{\alpha^{2}+\beta^{2}} \\ y_{1,2}=\frac{\theta \cdot \beta \pm \alpha \cdot \sqrt{\left(\alpha^{2}+\beta^{2}\right) \cdot L^{2}-\theta^{2}}}{\alpha^{2}+\beta^{2}} \\ x_{1,2}=\frac{\theta-\beta \cdot y}{\alpha}=\frac{\theta}{\alpha}-\frac{\beta}{\alpha} \cdot y_{1,2}\end{array}\right.$ 
For proper positioning of the point $D$ is chosen initially negative solution (if it does not correspond to the positive solution will reelect). This gives scalar parameters of point $D$ (relation 18):

$$
\begin{cases}y=\frac{\theta \cdot \beta-\alpha \cdot \sqrt{\left(\alpha^{2}+\beta^{2}\right) \cdot L^{2}-\theta^{2}}}{\alpha^{2}+\beta^{2}} & y_{D}=y+y_{S} \\ x=\frac{\theta-\beta \cdot y}{\alpha}=\frac{\theta}{\alpha}-\frac{\beta}{\alpha} \cdot y & x_{D}=x+x_{S} \\ \Rightarrow D\left(x_{D}, y_{D}, z_{D}\right) & \end{cases}
$$

From $(12,10,9)$ are selected the equations with that one write the system (19), so as to have the only unknown the scalar coordinates of the point $E$, namely: $x_{E}, y_{E}, z_{E}$ :

$$
\left\{\begin{array}{l}
\left(x_{E}-x_{S}\right) \cdot \alpha+\left(y_{E}-y_{S}\right) \cdot \beta+\left(z_{E}-z_{S}\right) \cdot \gamma=0 \\
\left(x_{E}-x_{S}\right)^{2}+\left(y_{E}-y_{S}\right)^{2}+\left(z_{E}-z_{S}\right)^{2}=R^{2} \\
\left(x_{E}-x_{D}\right)^{2}+\left(y_{E}-y_{D}\right)^{2}+\left(z_{E}-z_{D}\right)^{2}=3 \cdot R^{2}
\end{array}\right.
$$

To solve the system (19) must be linearized. One squares the last two relations system (19) and subtract the second from the third. Third relationship is obtained in the system (20) which is arranged at a more convenient form trapped in the system (21) together with the first relation from the system (19) also ordered correspondingly:

$$
\begin{aligned}
& \left\{\begin{array}{l}
x_{E}^{2}+x_{S}^{2}-2 \cdot x_{S} \cdot x_{E}+y_{E}^{2}+y_{S}^{2} \\
-2 \cdot y_{S} \cdot y_{E}+z_{E}^{2}+z_{S}^{2}-2 \cdot z_{S} \cdot z_{E}=R^{2} \\
x_{E}^{2}+x_{D}^{2}-2 \cdot x_{D} \cdot x_{E}+y_{E}^{2}+y_{D}^{2} \\
-2 \cdot y_{D} \cdot y_{E}+z_{E}^{2}+z_{D}^{2}-2 \cdot z_{D} \cdot z_{E}=3 \cdot R^{2} \\
------------------ \\
x_{D}^{2}-x_{S}^{2}+2 \cdot\left(x_{S}-x_{D}\right) \cdot x_{E}+y_{D}^{2}-y_{S}^{2} \\
+2 \cdot\left(y_{S}-y_{D}\right) \cdot y_{E}+z_{D}^{2}-z_{S}^{2}+ \\
+2 \cdot\left(z_{S}-z_{D}\right) \cdot z_{E}=2 \cdot R^{2}
\end{array}\right. \\
& \left\{\begin{array}{l}
2 \cdot\left(x_{S}-x_{D}\right) \cdot x_{E}+2 \cdot\left(y_{S}-y_{D}\right) \cdot y_{E}+2 \cdot\left(z_{S}-z_{D}\right) \cdot z_{E} \\
=2 \cdot R^{2}+x_{S}^{2}+y_{S}^{2}+z_{S}^{2}-x_{D}^{2}-y_{D}^{2}-z_{D}^{2} \\
\alpha \cdot x_{E}+\beta \cdot y_{E}+\gamma \cdot z_{E}=\alpha \cdot x_{S}+\beta \cdot y_{S}+\gamma \cdot z_{S}
\end{array}\right.
\end{aligned}
$$

From the second relation of the system (21) are explained $z_{E}$ (see expression 22), which is then introduced into the first relation of the system (21) thereby eliminating $z_{E}$ parameter and to give expression (23) linearly with $y_{E}$ in relation of $x_{E}$, where the coefficients $k_{1}, k_{2}$, are determined by the relationships of the system (24):

$$
\begin{aligned}
& z_{E}=\frac{\alpha}{\gamma} \cdot x_{S}+\frac{\beta}{\gamma} \cdot y_{S}+z_{S}-\frac{\alpha}{\gamma} \cdot x_{E}-\frac{\beta}{\gamma} \cdot y_{E} \\
& y_{E}=k_{1}+k_{2} \cdot x_{E} \\
& \left\{\begin{array}{l}
k_{1}=\left[2 \cdot R^{2}+x_{S}^{2}+y_{S}^{2}+z_{S}^{2}-x_{D}^{2}-y_{D}^{2}-z_{D}^{2}\right. \\
-2 \cdot\left(z_{S}-z_{D}\right) \cdot \frac{\alpha}{\gamma} \cdot x_{S}-2 \cdot\left(z_{S}-z_{D}\right) \cdot \frac{\beta}{\gamma} \cdot y_{S} \\
-2 \cdot\left(z_{S}-z_{D}\right) \cdot z_{S} \\
{\left[\begin{array}{l}
2 \cdot\left(y_{S}-y_{D}\right)-2 \cdot\left(z_{S}-z_{D}\right) \cdot \frac{\beta}{\gamma}
\end{array}\right]:} \\
k_{2}=\frac{\left(x_{D}-x_{S}\right)+\left(z_{S}-z_{D}\right) \cdot \frac{\alpha}{\gamma}}{\left(y_{S}-y_{D}\right)-\left(z_{S}-z_{D}\right) \cdot \frac{\beta}{\gamma}}
\end{array}\right]
\end{aligned}
$$

Replace now $y_{E}$ given by the relation (23) in the expression (22) and thus obtains a second linear relationship between parameters $z_{E}$ and $x_{E}$ (Equation 25), whose coefficients $k_{3}, k_{4}$, are given by the system (26):

$z_{E}=k_{3}-k_{4} \cdot x_{E}$

$\left\{\begin{array}{l}k_{3}=\frac{\alpha}{\gamma} \cdot x_{S}+\frac{\beta}{\gamma} \cdot y_{S}+z_{S}-\frac{\beta}{\gamma} \cdot k_{1} \\ k_{4}=\frac{\alpha}{\gamma}+\frac{\beta}{\gamma} \cdot k_{2}\end{array}\right.$

Relationships (23) and (25) are included simultaneously into the first relationship of the system (20) and in this mode one obtains an equation grade 2 in $x_{E}$ (expression 27), which is allocated to the form (28):

$$
\begin{aligned}
& \left\{\begin{array}{l}
x_{E}^{2}-2 \cdot x_{S} \cdot x_{E}+\left(k_{1}+k_{2} \cdot x_{E}\right)^{2}-2 \cdot y_{S} \cdot\left(k_{1}+k_{2} \cdot x_{E}\right) \\
+\left(k_{3}-k_{4} \cdot x_{E}\right)^{2}-2 \cdot z_{S} \cdot\left(k_{3}-k_{4} \cdot x_{E}\right) \\
=R^{2}-x_{S}^{2}-y_{S}^{2}-z_{S}^{2}
\end{array}\right. \\
& \left\{\begin{array}{l}
\left(1+k_{2}^{2}+k_{4}^{2}\right) \cdot x_{E}^{2}-2 \cdot\left(x_{S}-k_{1} \cdot k_{2}+k_{2} \cdot y_{S}+k_{3} \cdot k_{4}\right) \cdot x_{E} \\
+k_{1}^{2}-2 \cdot k_{1} \cdot y_{S}+k_{3}^{2}-2 \cdot k_{3} \cdot z_{S}-R^{2}+x_{S}^{2}+y_{S}^{2}+z_{S}^{2}=0
\end{array}\right.
\end{aligned}
$$

We denote now the coefficients of Equation 28 of second degree in $x_{E}$, with $a_{1}, b_{1}, c_{1}$, (see relation 29 ):

$$
\left\{\begin{array}{l}
a_{1}=1+k_{2}^{2}+k_{4}^{2} \\
b_{1} \equiv-\frac{b}{2}=x_{S}-k_{1} \cdot k_{2}+k_{2} \cdot y_{S}+k_{3} \cdot k_{4} \\
c_{1}=k_{1}^{2}-2 \cdot k_{1} \cdot y_{S}+k_{3}^{2}-2 \cdot k_{3} \cdot z_{S}-R^{2}+x_{S}^{2}+y_{S}^{2}+z_{S}^{2}
\end{array}\right.
$$

Equation 28 takes the simplified form (30) which supports real solutions (31): 


$$
\begin{aligned}
& a_{1} \cdot x_{E}^{2}-2 \cdot b_{1} \cdot x_{E}+c_{1}=0 \\
& x_{E_{1,2}}=\frac{b_{1} \pm \sqrt{b_{1}^{2}-a_{1} \cdot c_{1}}}{a_{1}}
\end{aligned}
$$

Choose the appropriate solution (the plus or the minus). Perhaps the solution would be negative.

One writes all scalar parameters of the point $E$, by relations (32):

$$
\left\{\begin{array}{l}
x_{E}=\frac{b_{1}}{a_{1}}-\sqrt{\left(\frac{b_{1}}{a_{1}}\right)^{2}-\frac{c_{1}}{a_{1}}} \\
y_{E}=k_{1}+k_{2} \cdot x_{E} \\
z_{E}=k_{3}-k_{4} \cdot x_{E}
\end{array}\right.
$$

Now we have to determine Cartesian coordinates (rectangular, scalar) of mobile point $F$. From initial systems $(12,10,9)$ we can choose to use four relationships (one of 12, one of 10 and two from 9), relations with that one writes the system (33):

$$
\left\{\begin{array}{l}
\left(x_{F}-x_{S}\right) \cdot \alpha+\left(y_{F}-y_{S}\right) \cdot \beta+\left(z_{F}-z_{S}\right) \cdot \gamma=0 \\
\left(x_{F}-x_{S}\right)^{2}+\left(y_{F}-y_{S}\right)^{2}+\left(z_{F}-z_{S}\right)^{2}=R^{2} \\
\left(x_{F}-x_{D}\right)^{2}+\left(y_{F}-y_{D}\right)^{2}+\left(z_{F}-z_{D}\right)^{2}=3 \cdot R^{2} \\
\left(x_{F}-x_{E}\right)^{2}+\left(y_{F}-y_{E}\right)^{2}+\left(z_{F}-z_{E}\right)^{2}=3 \cdot R^{2}
\end{array}\right.
$$

The squared last two relations of the system (33) as expressions obtained (34) gather resulting Equation 35, which is then arranged conveniently to the final form (36):

$$
\begin{aligned}
& \left\{\begin{array}{l}
x_{F}^{2}+x_{D}^{2}-2 \cdot x_{D} \cdot x_{F}+y_{F}^{2}+y_{D}^{2} \\
-2 \cdot y_{D} \cdot y_{F}+z_{F}^{2}+z_{D}^{2}-2 \cdot z_{D} \cdot z_{F}=3 \cdot R^{2} \\
x_{F}^{2}+x_{E}^{2}-2 \cdot x_{E} \cdot x_{F}+y_{F}^{2}+y_{E}^{2} \\
-2 \cdot y_{E} \cdot y_{F}+z_{F}^{2}+z_{E}^{2}-2 \cdot z_{E} \cdot z_{F}=3 \cdot R^{2}
\end{array}\right. \\
& \left\{\begin{array}{l}
x_{D}^{2}-x_{E}^{2}+2 \cdot\left(x_{E}-x_{D}\right) \cdot x_{F}+y_{D}^{2}-y_{E}^{2} \\
+2 \cdot\left(y_{E}-y_{D}\right) \cdot y_{F}+z_{D}^{2}-z_{E}^{2} \\
+2 \cdot\left(z_{E}-z_{D}\right) \cdot z_{F}=0
\end{array}\right. \\
& \left\{\begin{array}{l}
2 \cdot\left(x_{E}-x_{D}\right) \cdot x_{F}+2 \cdot\left(y_{E}-y_{D}\right) \cdot y_{F} \\
+2 \cdot\left(z_{E}-z_{D}\right) \cdot z_{F} \\
=x_{E}^{2}-x_{D}^{2}+y_{E}^{2}-y_{D}^{2}+z_{E}^{2}-z_{D}^{2}
\end{array}\right.
\end{aligned}
$$

Repeat all for the couple of equations the second and the third of the system of Equation 33; one obtains the two equations system (37), which added produce the expression (38) that are arranged conveniently in the expression (39):

$\left\{\begin{array}{l}x_{F}^{2}+x_{S}^{2}-2 \cdot x_{S} \cdot x_{F}+y_{F}^{2}+y_{S}^{2}- \\ -2 \cdot y_{S} \cdot y_{F}+z_{F}^{2}+z_{S}^{2}-2 \cdot z_{S} \cdot z_{F}=R^{2} \\ x_{F}^{2}+x_{D}^{2}-2 \cdot x_{D} \cdot x_{F}+y_{F}^{2}+y_{D}^{2}- \\ -2 \cdot y_{D} \cdot y_{F}+z_{F}^{2}+z_{D}^{2}-2 \cdot z_{D} \cdot z_{F}=3 \cdot R^{2}\end{array}\right.$

$\left\{\begin{array}{l}x_{D}^{2}-x_{S}^{2}+2 \cdot\left(x_{S}-x_{D}\right) \cdot x_{F}+y_{D}^{2}-y_{S}^{2} \\ +2 \cdot\left(y_{S}-y_{D}\right) \cdot y_{F}+z_{D}^{2}-z_{S}^{2} \\ +2 \cdot\left(z_{S}-z_{D}\right) \cdot z_{F}=2 \cdot R^{2}\end{array}\right.$

$\left\{\begin{array}{l}2 \cdot\left(x_{S}-x_{D}\right) \cdot x_{F}+2 \cdot\left(y_{S}-y_{D}\right) \cdot y_{F} \\ +2 \cdot\left(z_{S}-z_{D}\right) \cdot z_{F} \\ =2 \cdot R^{2}+x_{S}^{2}-x_{D}^{2}+y_{S}^{2}-y_{D}^{2}+z_{S}^{2}-z_{D}^{2}\end{array}\right.$

It retains the linear system (40) of three equations with three unknowns, where the three equations are (36), (39) and the first relation of the system (33):

$$
\left\{\begin{array}{l}
2\left(x_{E}-x_{D}\right) x_{F}+2\left(y_{E}-y_{D}\right) y_{F}+2\left(z_{E}-z_{D}\right) z_{F} \\
=x_{E}^{2}-x_{D}^{2}+y_{E}^{2}-y_{D}^{2}+z_{E}^{2}-z_{D}^{2} \\
2\left(x_{S}-x_{D}\right) x_{F}+2\left(y_{S}-y_{D}\right) y_{F}+2\left(z_{S}-z_{D}\right) z_{F} \\
=2 R^{2}+x_{S}^{2}-x_{D}^{2}+y_{S}^{2}-y_{D}^{2}+z_{S}^{2}-z_{D}^{2} \\
\alpha \cdot x_{F}+\beta \cdot y_{F}+\gamma \cdot z_{F}=\alpha \cdot x_{S}+\beta \cdot y_{S}+\gamma \cdot z_{S}
\end{array}\right.
$$

The system (40) is written as classical (41):

$\left\{\begin{array}{l}a_{11} \cdot x_{F}+a_{12} \cdot y_{F}+a_{13} \cdot z_{F}=b_{1} \\ a_{21} \cdot x_{F}+a_{22} \cdot y_{F}+a_{23} \cdot z_{F}=b_{2} \\ a_{31} \cdot x_{F}+a_{32} \cdot y_{F}+a_{33} \cdot z_{F}=b_{3}\end{array}\right.$

The coefficients of the system (41) are determined by the relations (42):

$\left\{\begin{array}{l}a_{11}=2 \cdot\left(x_{E}-x_{D}\right) ; \\ a_{12}=2 \cdot\left(y_{E}-y_{D}\right) ; \\ a_{13}=2 \cdot\left(z_{E}-z_{D}\right) ; \\ b_{1}=x_{E}^{2}-x_{D}^{2}+y_{E}^{2}-y_{D}^{2}+z_{E}^{2}-z_{D}^{2} ; \\ a_{21}=2 \cdot\left(x_{S}-x_{D}\right) ; \\ a_{22}=2 \cdot\left(y_{S}-y_{D}\right) ; \\ a_{23}=2 \cdot\left(z_{S}-z_{D}\right) ; \\ b_{2}=2 \cdot R^{2}+x_{S}^{2}-x_{D}^{2}+y_{S}^{2}-y_{D}^{2}+z_{S}^{2}-z_{D}^{2} ; \\ a_{31}=\alpha ; \quad a_{32}=\beta ; \quad a_{33}=\gamma ; \\ b_{3}=\alpha \cdot x_{S}+\beta \cdot y_{S}+\gamma \cdot z_{S}\end{array}\right.$ 
Determinants system (41) is determined by relations (43-46):

$$
\begin{aligned}
& \Delta=\left|\begin{array}{lll}
a_{11} & a_{12} & a_{13} \\
a_{21} & a_{22} & a_{23} \\
a_{31} & a_{32} & a_{33}
\end{array}\right| \\
& =a_{11} \cdot\left(a_{22} \cdot a_{33}-a_{23} \cdot a_{32}\right) \\
& +a_{12} \cdot\left(a_{23} \cdot a_{31}-a_{21} \cdot a_{33}\right) \\
& +a_{13} \cdot\left(a_{21} \cdot a_{32}-a_{22} \cdot a_{31}\right)
\end{aligned}
$$$$
\Delta_{x}=\left|\begin{array}{lll}
b_{1} & a_{12} & a_{13} \\
b_{2} & a_{22} & a_{23} \\
b_{3} & a_{32} & a_{33}
\end{array}\right|
$$$$
=b_{1} \cdot\left(a_{22} \cdot a_{33}-a_{23} \cdot a_{32}\right)
$$$$
+a_{12} \cdot\left(a_{23} \cdot b_{3}-b_{2} \cdot a_{33}\right)
$$$$
+a_{13} \cdot\left(b_{2} \cdot a_{32}-a_{22} \cdot b_{3}\right)
$$

$$
\Delta_{y}=\left|\begin{array}{lll}
a_{11} & b_{1} & a_{13} \\
a_{21} & b_{2} & a_{23} \\
a_{31} & b_{3} & a_{33}
\end{array}\right|
$$$$
=a_{11} \cdot\left(b_{2} \cdot a_{33}-a_{23} \cdot b_{3}\right)
$$$$
+b_{1} \cdot\left(a_{23} \cdot a_{31}-a_{21} \cdot a_{33}\right)
$$$$
+a_{13} \cdot\left(a_{21} \cdot b_{3}-b_{2} \cdot a_{31}\right)
$$

$$
\begin{aligned}
& \Delta_{z}=\left|\begin{array}{lll}
a_{11} & a_{12} & b_{1} \\
a_{21} & a_{22} & b_{2} \\
a_{31} & a_{32} & b_{3}
\end{array}\right| \\
& =a_{11} \cdot\left(a_{22} \cdot b_{3}-b_{2} \cdot a_{32}\right) \\
& +a_{12} \cdot\left(b_{2} \cdot a_{31}-a_{21} \cdot b_{3}\right) \\
& +b_{1} \cdot\left(a_{21} \cdot a_{32}-a_{22} \cdot a_{31}\right)
\end{aligned}
$$

System solutions are given by relations (47):

$$
\left\{\begin{array}{l}
x_{F}=\frac{\Delta_{x}}{\Delta} \\
y_{F}=\frac{\Delta_{y}}{\Delta} \\
z_{F}=\frac{\Delta_{z}}{\Delta}
\end{array}\right.
$$

With the known coordinates of the points $D, E, F$, imposed by the choice of position of the DEF plan and point $D$, one determines then the required length of the legs (see the relations 48):

$$
\left\{\begin{array}{l}
l_{1}=\sqrt{\left(x_{D}-x_{A}\right)^{2}+\left(y_{D}-y_{A}\right)^{2}+\left(z_{D}-z_{A}\right)^{2}} \\
l_{2}=\sqrt{\left(x_{D}-x_{B}\right)^{2}+\left(y_{D}-y_{B}\right)^{2}+\left(z_{D}-z_{B}\right)^{2}} \\
l_{3}=\sqrt{\left(x_{E}-x_{B}\right)^{2}+\left(y_{E}-y_{B}\right)^{2}+\left(z_{E}-z_{B}\right)^{2}} \\
l_{4}=\sqrt{\left(x_{E}-x_{C}\right)^{2}+\left(y_{E}-y_{C}\right)^{2}+\left(z_{E}-z_{C}\right)^{2}} \\
l_{5}=\sqrt{\left(x_{F}-x_{C}\right)^{2}+\left(y_{F}-y_{C}\right)^{2}+\left(z_{F}-z_{C}\right)^{2}} \\
l_{6}=\sqrt{\left(x_{F}-x_{A}\right)^{2}+\left(y_{F}-y_{A}\right)^{2}+\left(z_{F}-z_{A}\right)^{2}}
\end{array}\right.
$$

\section{Determining the Velocitie}

One knows: $\dot{x}_{S}, \dot{y}_{S}, \dot{z}_{S}, \dot{\alpha}, \dot{\beta}, \dot{\gamma}, \dot{z}_{D}$. Choose the relations (13) writhed here as (49) to start:

$$
\left\{\begin{array}{l}
\left(x_{D}-x_{S}\right) \cdot \alpha+\left(y_{D}-y_{S}\right) \cdot \beta=\left(z_{S}-z_{D}\right) \cdot \gamma \\
\left(x_{D}-x_{S}\right)^{2}+\left(y_{D}-y_{S}\right)^{2}=R^{2}-\left(z_{D}-z_{S}\right)^{2}
\end{array}\right.
$$

Relationships (49) derived in function of time give the expressions (50):

$$
\left\{\begin{array}{l}
\left(\dot{x}_{D}-\dot{x}_{S}\right) \cdot \alpha+\left(x_{D}-x_{S}\right) \cdot \dot{\alpha}+\left(\dot{y}_{D}-\dot{y}_{S}\right) \cdot \beta+\left(y_{D}-y_{S}\right) \cdot \dot{\beta} \\
=\left(\dot{z}_{S}-\dot{z}_{D}\right) \cdot \gamma+\left(z_{S}-z_{D}\right) \cdot \dot{\gamma} \\
2 \cdot\left(x_{D}-x_{S}\right) \cdot\left(\dot{x}_{D}-\dot{x}_{S}\right)+2 \cdot\left(y_{D}-y_{S}\right) \cdot\left(\dot{y}_{D}-\dot{y}_{S}\right) \\
=-2 \cdot\left(z_{D}-z_{S}\right) \cdot\left(\dot{z}_{D}-\dot{z}_{S}\right)
\end{array}\right.
$$

These are arranged in the form (51). There was thus obtained a linear system of two equations in two unknowns, identified by (52):

$$
\left\{\begin{array}{l}
\alpha \cdot \dot{x}_{D}+\beta \cdot \dot{y}_{D}=\alpha \cdot \dot{x}_{S}-\left(x_{D}-x_{S}\right) \cdot \dot{\alpha}+\beta \cdot \dot{y}_{S} \\
-\left(y_{D}-y_{S}\right) \cdot \dot{\beta}+\left(\dot{z}_{S}-\dot{z}_{D}\right) \cdot \gamma+\left(z_{S}-z_{D}\right) \cdot \dot{\gamma} \\
\left(x_{D}-x_{S}\right) \cdot \dot{x}_{D}+\left(y_{D}-y_{S}\right) \cdot \dot{y}_{D}=\left(x_{D}-x_{S}\right) \cdot \dot{x}_{S} \\
+\left(y_{D}-y_{S}\right) \cdot \dot{y}_{S}-\left(z_{D}-z_{S}\right) \cdot\left(\dot{z}_{D}-\dot{z}_{S}\right)
\end{array}\right.
$$

$$
\left\{\begin{array}{l}
a_{11} \cdot \dot{x}_{D}+a_{12} \cdot \dot{y}_{D}=b_{1} \\
a_{21} \cdot \dot{x}_{D}+a_{22} \cdot \dot{y}_{D}=b_{2} \\
a_{11}=\alpha ; \quad a_{12}=\beta ; \\
a_{21}=x_{D}-x_{S} ; \quad a_{22}=y_{D}-y_{S} ; \\
b_{1}=\alpha \cdot \dot{x}_{S}-\left(x_{D}-x_{S}\right) \cdot \dot{\alpha}+\beta \cdot \dot{y}_{S}- \\
-\left(y_{D}-y_{S}\right) \cdot \dot{\beta}+\left(\dot{z}_{S}-\dot{z}_{D}\right) \cdot \gamma+\left(z_{S}-z_{D}\right) \cdot \dot{\gamma} \\
b_{2}=\left(x_{D}-x_{S}\right) \cdot \dot{x}_{S}+\left(y_{D}-y_{S}\right) \cdot \dot{y}_{S}- \\
-\left(z_{D}-z_{S}\right) \cdot\left(\dot{z}_{D}-\dot{z}_{S}\right)
\end{array}\right.
$$

Determinant of the system (51-52) is written in Equation 53: 


$$
\begin{aligned}
& \Delta=\left|\begin{array}{ll}
a_{11} & a_{12} \\
a_{21} & a_{22}
\end{array}\right|=a_{11} \cdot a_{22}-a_{12} \cdot a_{21} \\
& =\alpha \cdot\left(y_{D}-y_{S}\right)-\beta \cdot\left(x_{D}-x_{S}\right)
\end{aligned}
$$

One calculates $\Delta_{x 1}$ with the relation (54) and $\dot{x}_{D}$ by the expression (55):

$$
\begin{aligned}
& \Delta_{x 1}=\left|\begin{array}{ll}
b_{1} & a_{12} \\
b_{2} & a_{22}
\end{array}\right|=b_{1} \cdot a_{22}-a_{12} \cdot b_{2} \\
& \dot{x}_{D}=\frac{\Delta_{x 1}}{\Delta}
\end{aligned}
$$

One calculates $\Delta_{y 1}$ with the relation (56) and $\dot{y}_{D}$ by the expression (57):

$$
\begin{aligned}
& \Delta_{y 1}=\left|\begin{array}{ll}
a_{11} & b_{1} \\
a_{21} & b_{2}
\end{array}\right|=a_{11} \cdot b_{2}-b_{1} \cdot a_{21} \\
& \dot{y}_{D}=\frac{\Delta_{y 1}}{\Delta}
\end{aligned}
$$

It is still write system (58), which is derived in function of time and in this mode taking the form (59):

$$
\begin{aligned}
& \left\{\begin{array}{l}
\left(x_{E}-x_{S}\right) \cdot \alpha+\left(y_{E}-y_{S}\right) \cdot \beta+\left(z_{E}-z_{S}\right) \cdot \gamma=0 \\
\left(x_{E}-x_{S}\right)^{2}+\left(y_{E}-y_{S}\right)^{2}+\left(z_{E}-z_{S}\right)^{2}=R^{2} \\
\left(x_{E}-x_{D}\right)^{2}+\left(y_{E}-y_{D}\right)^{2}+\left(z_{E}-z_{D}\right)^{2}=3 \cdot R^{2}
\end{array}\right. \\
& \left\{\begin{array}{l}
\left(\dot{x}_{E}-\dot{x}_{S}\right) \cdot \alpha+\left(x_{E}-x_{S}\right) \cdot \dot{\alpha}+\left(\dot{y}_{E}-\dot{y}_{S}\right) \cdot \beta \\
+\left(y_{E}-y_{S}\right) \cdot \dot{\beta}+\left(\dot{z}_{E}-\dot{z}_{S}\right) \cdot \gamma+\left(z_{E}-z_{S}\right) \cdot \dot{\gamma}=0 \\
2 \cdot\left(x_{E}-x_{S}\right) \cdot\left(\dot{x}_{E}-\dot{x}_{S}\right)+2 \cdot\left(y_{E}-y_{S}\right) \cdot\left(\dot{y}_{E}-\dot{y}_{S}\right) \\
+2 \cdot\left(z_{E}-z_{S}\right) \cdot\left(\dot{z}_{E}-\dot{z}_{S}\right)=0 \\
2 \cdot\left(x_{E}-x_{D}\right) \cdot\left(\dot{x}_{E}-\dot{x}_{D}\right)+2 \cdot\left(y_{E}-y_{D}\right) \cdot\left(\dot{y}_{E}-\dot{y}_{D}\right) \\
+2 \cdot\left(z_{E}-z_{D}\right) \cdot\left(\dot{z}_{E}-\dot{z}_{D}\right)=0
\end{array}\right.
\end{aligned}
$$

For solving the system (59) is allocated to the form (60), which is a linear system of three equations with three unknown level one, identified by formulas in the system (61):

$$
\left\{\begin{array}{l}
\alpha \cdot \dot{x}_{E}+\beta \cdot \dot{y}_{E}+\gamma \cdot \dot{z}_{E}=\alpha \cdot \dot{x}_{S}-\left(x_{E}-x_{S}\right) \cdot \dot{\alpha} \\
+\beta \cdot \dot{y}_{S}-\left(y_{E}-y_{S}\right) \cdot \dot{\beta}+\gamma \cdot \dot{z}_{S}-\left(z_{E}-z_{S}\right) \cdot \dot{\gamma} \\
\left(x_{E}-x_{S}\right) \cdot \dot{x}_{E}+\left(y_{E}-y_{S}\right) \cdot \dot{y}_{E}+\left(z_{E}-z_{S}\right) \cdot \dot{z}_{E} \\
=\left(x_{E}-x_{S}\right) \cdot \dot{x}_{S}+\left(y_{E}-y_{S}\right) \cdot \dot{y}_{S}+\left(z_{E}-z_{S}\right) \cdot \dot{z}_{S} \\
\left(x_{E}-x_{D}\right) \cdot \dot{x}_{E}+\left(y_{E}-y_{D}\right) \cdot \dot{y}_{E}+\left(z_{E}-z_{D}\right) \cdot \dot{z}_{E} \\
=\left(x_{E}-x_{D}\right) \cdot \dot{x}_{D}+\left(y_{E}-y_{D}\right) \cdot \dot{y}_{D}+\left(z_{E}-z_{D}\right) \cdot \dot{z}_{D}
\end{array}\right.
$$

$$
\left\{\begin{array}{l}
c_{11} \cdot \dot{x}_{E}+c_{12} \cdot \dot{y}_{E}+c_{13} \cdot \dot{z}_{E}=c_{1} \\
c_{21} \cdot \dot{x}_{E}+c_{22} \cdot \dot{y}_{E}+c_{23} \cdot \dot{z}_{E}=c_{2} \\
c_{31} \cdot \dot{x}_{E}+c_{32} \cdot \dot{y}_{E}+c_{33} \cdot \dot{z}_{E}=c_{3} \\
c_{11}=\alpha ; \quad c_{12}=\beta ; \quad c_{13}=\gamma ; \\
c_{1}=\alpha \cdot \dot{x}_{S}-\left(x_{E}-x_{S}\right) \cdot \dot{\alpha}+\beta \cdot \dot{y}_{S}- \\
-\left(y_{E}-y_{S}\right) \cdot \dot{\beta}+\gamma \cdot \dot{z}_{S}-\left(z_{E}-z_{S}\right) \cdot \dot{\gamma} \\
c_{21}=x_{E}-x_{S} ; \quad c_{22}=y_{E}-y_{S} ; \quad c_{23}=z_{E}-z_{s} ; \\
c_{2}=\left(x_{E}-x_{S}\right) \cdot \dot{x}_{S}+\left(y_{E}-y_{S}\right) \cdot \dot{y}_{S}+\left(z_{E}-z_{S}\right) \cdot \dot{z}_{S} \\
c_{31}=x_{E}-x_{D} ; \quad c_{32}=y_{E}-y_{D} ; \quad c_{33}=z_{E}-z_{D} ; \\
c_{3}=\left(x_{E}-x_{D}\right) \cdot \dot{x}_{D}+\left(y_{E}-y_{D}\right) \cdot \dot{y}_{D}+\left(z_{E}-z_{D}\right) \cdot \dot{z}_{D}
\end{array}\right.
$$

The main determinant of the system (61) is given by relations (62):

$$
\left\{\begin{array}{l}
\Delta^{(c)}=\left|\begin{array}{lll}
c_{11} & c_{12} & c_{13} \\
c_{21} & c_{22} & c_{23} \\
c_{31} & c_{32} & c_{33}
\end{array}\right|=c_{11} \cdot\left(c_{22} \cdot c_{33}-c_{23} \cdot c_{32}\right) \\
-c_{12} \cdot\left(c_{21} \cdot c_{33}-c_{23} \cdot c_{31}\right)+c_{13} \cdot\left(c_{21} \cdot c_{32}-c_{22} \cdot c_{31}\right) \\
\Delta^{(c)}=\alpha \cdot\left[\left(y_{E}-y_{S}\right) \cdot\left(z_{E}-z_{D}\right)-\left(z_{E}-z_{S}\right) \cdot\left(y_{E}-y_{D}\right)\right] \\
-\beta \cdot\left[\left(x_{E}-x_{S}\right) \cdot\left(z_{E}-z_{D}\right)-\left(z_{E}-z_{S}\right) \cdot\left(x_{E}-x_{D}\right)\right] \\
+\gamma \cdot\left[\left(x_{E}-x_{S}\right) \cdot\left(y_{E}-y_{D}\right)-\left(y_{E}-y_{S}\right) \cdot\left(x_{E}-x_{D}\right)\right]
\end{array}\right.
$$

Determinant first scalar speed is calculated with Equation 63:

$$
\left\{\begin{array}{l}
\Delta_{x}^{(c)}=\left|\begin{array}{lll}
c_{1} & c_{12} & c_{13} \\
c_{2} & c_{22} & c_{23} \\
c_{3} & c_{32} & c_{33}
\end{array}\right|=c_{1} \cdot\left(c_{22} \cdot c_{33}-c_{23} \cdot c_{32}\right) \\
-c_{12} \cdot\left(c_{2} \cdot c_{33}-c_{23} \cdot c_{3}\right)+c_{13} \cdot\left(c_{2} \cdot c_{32}-c_{22} \cdot c_{3}\right)
\end{array}\right.
$$

First scalar velocity $\dot{x}_{E}$ is determined by the expression (64):

$\dot{x}_{E}=\frac{\Delta_{x}^{(c)}}{\Delta^{(c)}}$

The determinant of the second scalar speed is calculated by Equation 65 :

$$
\left\{\begin{array}{l}
\Delta_{y}^{(c)}=\left|\begin{array}{lll}
c_{11} & c_{1} & c_{13} \\
c_{21} & c_{2} & c_{23} \\
c_{31} & c_{3} & c_{33}
\end{array}\right|=c_{11} \cdot\left(c_{2} \cdot c_{33}-c_{23} \cdot c_{3}\right) \\
-c_{1} \cdot\left(c_{21} \cdot c_{33}-c_{23} \cdot c_{31}\right)+c_{13} \cdot\left(c_{21} \cdot c_{3}-c_{2} \cdot c_{31}\right)
\end{array}\right.
$$


The second scalar velocity $\dot{y}_{E}$ is determined by the expression (66):

$\dot{y}_{E}=\frac{\Delta_{y}^{(c)}}{\Delta^{(c)}}$

The determinant of the third scalar speed is calculated with the relation (67):

$$
\left\{\begin{array}{l}
\Delta_{z}^{(c)}=\left|\begin{array}{lll}
c_{11} & c_{12} & c_{1} \\
c_{21} & c_{22} & c_{2} \\
c_{31} & c_{32} & c_{3}
\end{array}\right|=c_{11} \cdot\left(c_{22} \cdot c_{3}-c_{2} \cdot c_{32}\right) \\
-c_{12} \cdot\left(c_{21} \cdot c_{3}-c_{2} \cdot c_{31}\right)+c_{1} \cdot\left(c_{21} \cdot c_{32}-c_{22} \cdot c_{31}\right)
\end{array}\right.
$$

The third scalar velocity $\dot{z}_{E}$ is determined by the expression (68):

$\dot{z}_{E}=\frac{\Delta_{z}^{(c)}}{\Delta^{(c)}}$

For scalar velocities of the last floating point $\mathrm{F}$ one starts from the positions system known (69), which is derived by time and results the system (70):

$$
\begin{aligned}
& \left\{\begin{array}{l}
\left(x_{F}-x_{S}\right) \cdot \alpha+\left(y_{F}-y_{S}\right) \cdot \beta+\left(z_{F}-z_{S}\right) \cdot \gamma=0 \\
\left(x_{F}-x_{S}\right)^{2}+\left(y_{F}-y_{S}\right)^{2}+\left(z_{F}-z_{S}\right)^{2}=R^{2} \\
\left(x_{F}-x_{D}\right)^{2}+\left(y_{F}-y_{D}\right)^{2}+\left(z_{F}-z_{D}\right)^{2}=3 \cdot R^{2}
\end{array}\right. \\
& \left\{\begin{array}{l}
\left(\dot{x}_{F}-\dot{x}_{S}\right) \cdot \alpha+\left(x_{F}-x_{S}\right) \cdot \dot{\alpha}+\left(\dot{y}_{F}-\dot{y}_{S}\right) \cdot \beta \\
+\left(y_{F}-y_{S}\right) \cdot \dot{\beta}+\left(\dot{z}_{F}-\dot{z}_{S}\right) \cdot \gamma+\left(z_{F}-z_{S}\right) \cdot \dot{\gamma}=0 \\
2 \cdot\left(x_{F}-x_{S}\right) \cdot\left(\dot{x}_{F}-\dot{x}_{S}\right)+2 \cdot\left(y_{F}-y_{S}\right) \cdot\left(\dot{y}_{F}-\dot{y}_{S}\right) \\
+2 \cdot\left(z_{F}-z_{S}\right) \cdot\left(\dot{z}_{F}-\dot{z}_{S}\right)=0 \\
2 \cdot\left(x_{F}-x_{D}\right) \cdot\left(\dot{x}_{F}-\dot{x}_{D}\right)+2 \cdot\left(y_{F}-y_{D}\right) \cdot\left(\dot{y}_{F}-\dot{y}_{D}\right) \\
+2 \cdot\left(z_{F}-z_{D}\right) \cdot\left(\dot{z}_{F}-\dot{z}_{D}\right)=0
\end{array}\right.
\end{aligned}
$$

The system (70) is arranged in the form (71) which is a linear system of three equations with three unknowns first degree, whose equations are identified by (72) and whose parameters are written in the form (73):

$$
\left\{\begin{array}{l}
\alpha \cdot \dot{x}_{F}+\beta \cdot \dot{y}_{F}+\gamma \cdot \dot{z}_{F}=\alpha \cdot \dot{x}_{S}+\beta \cdot \dot{y}_{S}+\gamma \cdot \dot{z}_{S} \\
-\left(x_{F}-x_{S}\right) \cdot \dot{\alpha}-\left(y_{F}-y_{S}\right) \cdot \dot{\beta}-\left(z_{F}-z_{S}\right) \cdot \dot{\gamma} \\
\left(x_{F}-x_{S}\right) \cdot \dot{x}_{F}+\left(y_{F}-y_{S}\right) \cdot \dot{y}_{F}+\left(z_{F}-z_{S}\right) \cdot \dot{z}_{F} \\
=\left(x_{F}-x_{S}\right) \cdot \dot{x}_{S}+\left(y_{F}-y_{S}\right) \cdot \dot{y}_{S}+\left(z_{F}-z_{S}\right) \cdot \dot{z}_{S} \\
\left(x_{F}-x_{D}\right) \cdot \dot{x}_{F}+\left(y_{F}-y_{D}\right) \cdot \dot{y}_{F}+\left(z_{F}-z_{D}\right) \cdot \dot{z}_{F} \\
=\left(x_{F}-x_{D}\right) \cdot \dot{x}_{D}+\left(y_{F}-y_{D}\right) \cdot \dot{y}_{D}+\left(z_{F}-z_{D}\right) \cdot \dot{z}_{D}
\end{array}\right.
$$

$$
\left\{\begin{array}{l}
d_{11} \cdot \dot{x}_{F}+d_{12} \cdot \dot{y}_{F}+d_{13} \cdot \dot{z}_{F}=d_{1} \\
d_{21} \cdot \dot{x}_{F}+d_{22} \cdot \dot{y}_{F}+d_{23} \cdot \dot{z}_{F}=d_{2} \\
d_{31} \cdot \dot{x}_{F}+d_{32} \cdot \dot{y}_{F}+d_{33} \cdot \dot{z}_{F}=d_{3}
\end{array}\right.
$$

$$
\left\{\begin{array}{l}
d_{11}=\alpha ; \quad d_{12}=\beta ; \quad d_{13}=\gamma ; \\
d_{1}=\alpha \cdot \dot{x}_{S}+\beta \cdot \dot{y}_{S}+\gamma \cdot \dot{z}_{S}-\left(x_{F}-x_{S}\right) \cdot \dot{\alpha} \\
-\left(y_{F}-y_{S}\right) \cdot \dot{\beta}-\left(z_{F}-z_{S}\right) \cdot \dot{\gamma} ; \\
d_{21}=x_{F}-x_{S} ; \quad d_{22}=y_{F}-y_{S} ; \quad d_{23}=z_{F}-z_{S} ; \\
d_{2}=\left(x_{F}-x_{S}\right) \cdot \dot{x}_{S}+\left(y_{F}-y_{S}\right) \cdot \dot{y}_{S}+\left(z_{F}-z_{S}\right) \cdot \dot{z}_{S} \\
d_{31}=x_{F}-x_{D} ; \quad d_{32}=y_{F}-y_{D} ; \quad d_{33}=z_{F}-z_{D} ; \\
d_{3}=\left(x_{F}-x_{D}\right) \cdot \dot{x}_{D}+\left(y_{F}-y_{D}\right) \cdot \dot{y}_{D}+\left(z_{F}-z_{D}\right) \cdot \dot{z}_{D}
\end{array}\right.
$$

The four determinants of the system are written in Equation 74-77, the primary determinant being given even by relation (74):

$\left\{\begin{array}{l}\Delta^{(d)}=\left|\begin{array}{lll}d_{11} & d_{12} & d_{13} \\ d_{21} & d_{22} & d_{23} \\ d_{31} & d_{32} & d_{33}\end{array}\right|=d_{11} \cdot\left(d_{22} \cdot d_{33}-d_{23} \cdot d_{32}\right) \\ -d_{12} \cdot\left(d_{21} \cdot d_{33}-d_{23} \cdot d_{31}\right)+d_{13} \cdot\left(d_{21} \cdot d_{32}-d_{22} \cdot d_{31}\right)\end{array}\right.$

$\left\{\begin{array}{l}\Delta_{x}^{(d)}=\left|\begin{array}{lll}d_{1} & d_{12} & d_{13} \\ d_{2} & d_{22} & d_{23} \\ d_{3} & d_{32} & d_{33}\end{array}\right|=d_{1} \cdot\left(d_{22} \cdot d_{33}-d_{23} \cdot d_{32}\right) \\ -d_{12} \cdot\left(d_{2} \cdot d_{33}-d_{3} \cdot d_{23}\right)+d_{13} \cdot\left(d_{2} \cdot d_{32}-d_{3} \cdot d_{22}\right)\end{array}\right.$

$\left\{\begin{array}{l}\Delta_{y}^{(d)}=\left|\begin{array}{lll}d_{11} & d_{1} & d_{13} \\ d_{21} & d_{2} & d_{23} \\ d_{31} & d_{3} & d_{33}\end{array}\right|=d_{11} \cdot\left(d_{2} \cdot d_{33}-d_{3} \cdot d_{23}\right) \\ -d_{1} \cdot\left(d_{21} \cdot d_{33}-d_{23} \cdot d_{31}\right)+d_{13} \cdot\left(d_{21} \cdot d_{3}-d_{2} \cdot d_{31}\right)\end{array}\right.$

$$
\left\{\begin{array}{l}
\Delta_{z}^{(d)}=\left|\begin{array}{lll}
d_{11} & d_{12} & d_{1} \\
d_{21} & d_{22} & d_{2} \\
d_{31} & d_{32} & d_{3}
\end{array}\right|=d_{11} \cdot\left(d_{22} \cdot d_{3}-d_{2} \cdot d_{32}\right) \\
-d_{12} \cdot\left(d_{21} \cdot d_{3}-d_{2} \cdot d_{31}\right)+d_{1} \cdot\left(d_{21} \cdot d_{32}-d_{22} \cdot d_{31}\right)
\end{array}\right.
$$

Gear system scalar solutions are obtained by using the relations (78):

$$
\left\{\dot{x}_{F}=\frac{\Delta_{x}^{(d)}}{\Delta^{(d)}} ; \quad \dot{y}_{F}=\frac{\Delta_{y}^{(d)}}{\Delta^{(d)}} ; \quad \dot{z}_{F}=\frac{\Delta_{z}^{(d)}}{\Delta^{(d)}}\right.
$$

Mobile plan speeds (upper) being determined, one can move to the final stage in which will be determined linear 
speeds of the six engines couplers (the translation). Must to write first relations of positions (79):

$$
\left\{\begin{array}{l}
l_{1}^{2}=\left(x_{D}-x_{A}\right)^{2}+\left(y_{D}-y_{A}\right)^{2}+\left(z_{D}-z_{A}\right)^{2} \\
l_{2}^{2}=\left(x_{D}-x_{B}\right)^{2}+\left(y_{D}-y_{B}\right)^{2}+\left(z_{D}-z_{B}\right)^{2} \\
l_{3}^{2}=\left(x_{E}-x_{B}\right)^{2}+\left(y_{E}-y_{B}\right)^{2}+\left(z_{E}-z_{B}\right)^{2} \\
l_{4}^{2}=\left(x_{E}-x_{C}\right)^{2}+\left(y_{E}-y_{C}\right)^{2}+\left(z_{E}-z_{C}\right)^{2} \\
l_{5}^{2}=\left(x_{F}-x_{C}\right)^{2}+\left(y_{F}-y_{C}\right)^{2}+\left(z_{F}-z_{C}\right)^{2} \\
l_{6}^{2}=\left(x_{F}-x_{A}\right)^{2}+\left(y_{F}-y_{A}\right)^{2}+\left(z_{F}-z_{A}\right)^{2}
\end{array}\right.
$$

Relations system (79) it is derived by time and one obtains the expressions system (80), from that is explained the linear velocities of the motor elements (81):

$$
\begin{aligned}
& \left\{\begin{array}{l}
2 \cdot l_{1} \cdot \dot{l}_{1}=2 \cdot\left(x_{D}-x_{A}\right) \cdot \dot{x}_{D}+2 \cdot\left(y_{D}-y_{A}\right) \cdot \dot{y}_{D}+2 \cdot\left(z_{D}-z_{A}\right) \cdot \dot{z}_{D} \\
2 \cdot l_{2} \cdot \dot{l}_{2}=2 \cdot\left(x_{D}-x_{B}\right) \cdot \dot{x}_{D}+2 \cdot\left(y_{D}-y_{B}\right) \cdot \dot{y}_{D}+2 \cdot\left(z_{D}-z_{B}\right) \cdot \dot{z}_{D} \\
2 \cdot l_{3} \cdot \dot{l}_{3}=2 \cdot\left(x_{E}-x_{B}\right) \cdot \dot{x}_{E}+2 \cdot\left(y_{E}-y_{B}\right) \cdot \dot{y}_{E}+2 \cdot\left(z_{E}-z_{B}\right) \cdot \dot{z}_{E} \\
2 \cdot l_{4} \cdot \dot{l}_{4}=2 \cdot\left(x_{E}-x_{C}\right) \cdot \dot{x}_{E}+2 \cdot\left(y_{E}-y_{C}\right) \cdot \dot{y}_{E}+2 \cdot\left(z_{E}-z_{C}\right) \cdot \dot{z}_{E} \\
2 \cdot l_{5} \cdot \dot{l}_{5}=2 \cdot\left(x_{F}-x_{C}\right) \cdot \dot{x}_{F}+2 \cdot\left(y_{F}-y_{C}\right) \cdot \dot{y}_{F}+2 \cdot\left(z_{F}-z_{C}\right) \cdot \dot{z}_{F} \\
2 \cdot l_{6} \cdot \dot{l}_{6}=2 \cdot\left(x_{F}-x_{A}\right) \cdot \dot{x}_{F}+2 \cdot\left(y_{F}-y_{A}\right) \cdot \dot{y}_{F}+2 \cdot\left(z_{F}-z_{A}\right) \cdot \dot{z}_{F}
\end{array}\right. \\
& \left\{\begin{array}{l}
\dot{l}_{1}=\frac{\left(x_{D}-x_{A}\right) \cdot \dot{x}_{D}+\left(y_{D}-y_{A}\right) \cdot \dot{y}_{D}+\left(z_{D}-z_{A}\right) \cdot \dot{z}_{D}}{l_{1}} \\
i_{2}=\frac{\left(x_{D}-x_{B}\right) \cdot \dot{x}_{D}+\left(y_{D}-y_{B}\right) \cdot \dot{y}_{D}+\left(z_{D}-z_{B}\right) \cdot \dot{z}_{D}}{l_{3}} \\
i_{3}=\frac{\left(x_{E}-x_{B}\right) \cdot \dot{x}_{E}+\left(y_{E}-y_{B}\right) \cdot \dot{y}_{E}+\left(z_{E}-z_{B}\right) \cdot \dot{z}_{E}}{l_{3}} \\
i_{4}=\frac{\left(x_{E}-x_{C}\right) \cdot \dot{x}_{E}+\left(y_{E}-y_{C}\right) \cdot \dot{y}_{E}+\left(z_{E}-z_{C}\right) \cdot \dot{z}_{E}}{l_{4}} \\
i_{5}=\frac{\left(x_{F}-x_{C}\right) \cdot \dot{x}_{F}+\left(y_{F}-y_{C}\right) \cdot \dot{y}_{F}+\left(z_{F}-z_{C}\right) \cdot \dot{z}_{F}}{l_{5}} \\
i_{6}=\frac{\left(x_{F}-x_{A}\right) \cdot \dot{x}_{F}+\left(y_{F}-y_{A}\right) \cdot \dot{y}_{F}+\left(z_{F}-z_{A}\right) \cdot \dot{z}_{F}}{l_{6}}
\end{array}\right.
\end{aligned}
$$

\section{Determining the Accelerations}

One knows: $\ddot{x}_{S}, \ddot{y}_{S}, \ddot{z}_{S}, \ddot{\alpha}, \ddot{\beta}, \ddot{\gamma}, \ddot{z}_{D}$.

One starts from the velocities relations (82), arranged in the form (83):

$$
\begin{aligned}
& \left\{\begin{array}{l}
\left(\dot{x}_{D}-\dot{x}_{S}\right) \cdot \alpha+\left(x_{D}-x_{S}\right) \cdot \dot{\alpha}+\left(\dot{y}_{D}-\dot{y}_{S}\right) \cdot \beta \\
+\left(y_{D}-y_{S}\right) \cdot \dot{\beta}=\left(\dot{z}_{S}-\dot{z}_{D}\right) \cdot \gamma+\left(z_{S}-z_{D}\right) \cdot \dot{\gamma} \\
\left(x_{D}-x_{S}\right) \cdot\left(\dot{x}_{D}-\dot{x}_{S}\right)+\left(y_{D}-y_{S}\right) \cdot\left(\dot{y}_{D}-\dot{y}_{S}\right) \\
=-\left(z_{D}-z_{S}\right) \cdot\left(\dot{z}_{D}-\dot{z}_{S}\right)
\end{array}\right. \\
& \left\{\begin{array}{l}
\alpha \cdot \dot{x}_{D}+\beta \cdot \dot{y}_{D}=\alpha \cdot \dot{x}_{S}-\left(x_{D}-x_{S}\right) \cdot \dot{\alpha}+\beta \cdot \dot{y}_{S} \\
-\left(y_{D}-y_{S}\right) \cdot \dot{\beta}+\left(\dot{z}_{S}-\dot{z}_{D}\right) \cdot \gamma+\left(z_{S}-z_{D}\right) \cdot \dot{\gamma} \\
\left(x_{D}-x_{S}\right) \cdot \dot{x}_{D}+\left(y_{D}-y_{S}\right) \cdot \dot{y}_{D}=\left(x_{D}-x_{S}\right) \cdot \dot{x}_{S} \\
+\left(y_{D}-y_{S}\right) \cdot \dot{y}_{S}-\left(z_{D}-z_{S}\right) \cdot\left(\dot{z}_{D}-\dot{z}_{S}\right)
\end{array}\right.
\end{aligned}
$$

Expressions (83) are derived by time and one obtains the accelerations system (84) which can be arranged to the form (85):

$$
\begin{aligned}
& \left\{\begin{array}{l}
\dot{\alpha} \cdot \dot{x}_{D}+\alpha \cdot \ddot{x}_{D}+\dot{\beta} \cdot \dot{y}_{D}+\beta \cdot \ddot{y}_{D}=\dot{\alpha} \cdot \dot{x}_{S}+\alpha \cdot \ddot{x}_{S} \\
-\left(\dot{x}_{D}-\dot{x}_{S}\right) \cdot \dot{\alpha}-\left(x_{D}-x_{S}\right) \cdot \ddot{\alpha}+\dot{\beta} \cdot \dot{y}_{S}+\beta \cdot \ddot{y}_{S} \\
-\left(\dot{y}_{D}-\dot{y}_{S}\right) \cdot \dot{\beta}-\left(y_{D}-y_{S}\right) \cdot \ddot{\beta}+\left(\ddot{z}_{S}-\ddot{z}_{D}\right) \cdot \gamma \\
+\left(\dot{z}_{S}-\dot{z}_{D}\right) \cdot \dot{\gamma}+\left(\dot{z}_{S}-\dot{z}_{D}\right) \cdot \dot{\gamma}+\left(z_{S}-z_{D}\right) \cdot \ddot{\gamma} \\
\left(\dot{x}_{D}-\dot{x}_{S}\right) \cdot \dot{x}_{D}+\left(x_{D}-x_{S}\right) \cdot \ddot{x}_{D}+\left(\dot{y}_{D}-\dot{y}_{S}\right) \cdot \dot{y}_{D} \\
\left(y_{D}-y_{S}\right) \cdot \ddot{y}_{D}=\left(\dot{x}_{D}-\dot{x}_{S}\right) \cdot \dot{x}_{S}+\left(x_{D}-x_{S}\right) \cdot \ddot{x}_{S} \\
+\left(\dot{y}_{D}-\dot{y}_{S}\right) \cdot \dot{y}_{S}+\left(y_{D}-y_{S}\right) \cdot \ddot{y}_{S} \\
-\left(\dot{z}_{D}-\dot{z}_{S}\right)^{2}-\left(z_{D}-z_{S}\right) \cdot\left(\ddot{z}_{D}-\ddot{z}_{S}\right)
\end{array}\right. \\
& \left\{\begin{array}{l}
\alpha \cdot \ddot{x}_{D}+\beta \cdot \ddot{y}_{D}=2 \cdot \dot{\alpha} \cdot\left(\dot{x}_{S}-\dot{x}_{D}\right)+2 \cdot \dot{\beta} \cdot\left(\dot{y}_{S}-\dot{y}_{D}\right) \\
+\alpha \cdot \ddot{x}_{S}+\beta \cdot \ddot{y}_{S}+\left(x_{S}-x_{D}\right) \cdot \ddot{\alpha}+\left(y_{S}-y_{D}\right) \cdot \ddot{\beta} \\
+\left(\ddot{z}_{S}-\ddot{z}_{D}\right) \cdot \gamma+2 \cdot\left(\dot{z}_{S}-\dot{z}_{D}\right) \cdot \dot{\gamma}+\left(z_{S}-z_{D}\right) \cdot \ddot{\gamma} \\
\left(x_{D}-x_{S}\right) \cdot \ddot{x}_{D}+\left(y_{D}-y_{S}\right) \cdot \ddot{y}_{D}=-\left(\dot{x}_{D}-\dot{x}_{S}\right)^{2} \\
-\left(\dot{y}_{D}-\dot{y}_{S}\right)^{2}-\left(\dot{z}_{D}-\dot{z}_{S}\right)^{2}+\left(x_{D}-x_{S}\right) \cdot \ddot{x}_{S} \\
+\left(y_{D}-y_{S}\right) \cdot \ddot{y}_{S}-\left(z_{D}-z_{S}\right) \cdot\left(\ddot{z}_{D}-\ddot{z}_{S}\right)
\end{array}\right.
\end{aligned}
$$

One can identify the linear system of two equations with two unknowns (86) with coefficients (87) and solutions (88):

$\left\{\begin{array}{l}a_{11} \cdot \ddot{x}_{D}+a_{12} \cdot \ddot{y}_{D}=f_{1} \\ a_{21} \cdot \ddot{x}_{D}+a_{22} \cdot \ddot{y}_{D}=f_{2}\end{array}\right.$

$\left\{\begin{array}{l}a_{11}=\alpha ; \quad a_{12}=\beta ; \quad a_{21}=x_{D}-x_{S} ; \quad a_{22}=y_{D}-y_{S} ; \\ f_{1}=2 \cdot\left[\dot{\alpha} \cdot\left(\dot{x}_{S}-\dot{x}_{D}\right)+\dot{\beta} \cdot\left(\dot{y}_{S}-\dot{y}_{D}\right)+\dot{\gamma} \cdot\left(\dot{z}_{S}-\dot{z}_{D}\right)\right]+\alpha \cdot \ddot{x}_{S}+\beta \cdot \ddot{y}_{S} \\ +\gamma \cdot\left(\ddot{z}_{S}-\ddot{z}_{D}\right)+\left(x_{S}-x_{D}\right) \cdot \ddot{\alpha}+\left(y_{S}-y_{D}\right) \cdot \ddot{\beta}+\left(z_{S}-z_{D}\right) \cdot \ddot{\gamma} \\ f_{2}=-\left(\dot{x}_{D}-\dot{x}_{S}\right)^{2}-\left(\dot{y}_{D}-\dot{y}_{S}\right)^{2}-\left(\dot{z}_{D}-\dot{z}_{S}\right)^{2}+ \\ +\left(x_{D}-x_{S}\right) \cdot \ddot{x}_{S}+\left(y_{D}-y_{S}\right) \cdot \ddot{y}_{S}-\left(z_{D}-z_{S}\right) \cdot\left(\ddot{z}_{D}-\ddot{z}_{S}\right)\end{array}\right.$

$\left\{\begin{array}{l}\Delta_{f}=\left|\begin{array}{ll}a_{11} & a_{12} \\ a_{21} & a_{22}\end{array}\right|=a_{11} \cdot a_{22}-a_{12} \cdot a_{21} \\ \Delta_{x D 2}=\left|\begin{array}{ll}f_{1} & a_{12} \\ f_{2} & a_{22}\end{array}\right|=f_{1} \cdot a_{22}-f_{2} \cdot a_{12} \\ \Delta_{y D 2}=\left|\begin{array}{ll}a_{11} & f_{1} \\ a_{21} & f_{2}\end{array}\right|=f_{2} \cdot a_{11}-f_{1} \cdot a_{21} \\ \ddot{x}_{D}=\frac{\Delta_{x D 2}}{\Delta_{f}} ; \quad \ddot{y}_{D}=\frac{\Delta_{y D 2}}{\Delta_{f}}\end{array}\right.$

The velocities system (89) is derived by time and one obtains the accelerations relations (90), which are arranged to the form (91): 


$$
\begin{aligned}
& \left\{\begin{array}{l}
c_{11} \cdot \dot{x}_{E}+c_{12} \cdot \dot{y}_{E}+c_{13} \cdot \dot{z}_{E}=c_{1} \\
c_{21} \cdot \dot{x}_{E}+c_{22} \cdot \dot{y}_{E}+c_{23} \cdot \dot{z}_{E}=c_{2} \\
c_{31} \cdot \dot{x}_{E}+c_{32} \cdot \dot{y}_{E}+c_{33} \cdot \dot{z}_{E}=c_{3}
\end{array}\right. \\
& \left\{\begin{array}{l}
\dot{c}_{11} \cdot \dot{x}_{E}+\dot{c}_{12} \cdot \dot{y}_{E}+\dot{c}_{13} \cdot \dot{z}_{E}+c_{11} \cdot \ddot{x}_{E} \\
+c_{12} \cdot \ddot{y}_{E}+c_{13} \cdot \ddot{z}_{E}=\dot{c}_{1} \\
\dot{c}_{21} \cdot \dot{x}_{E}+\dot{c}_{22} \cdot \dot{y}_{E}+\dot{c}_{23} \cdot \dot{z}_{E}+c_{21} \cdot \ddot{x}_{E} \\
+c_{22} \cdot \ddot{y}_{E}+c_{23} \cdot \ddot{z}_{E}=\dot{c}_{2} \\
\dot{c}_{31} \cdot \dot{x}_{E}+\dot{c}_{32} \cdot \dot{y}_{E}+\dot{c}_{33} \cdot \dot{z}_{E}+c_{31} \cdot \ddot{x}_{E} \\
+c_{32} \cdot \ddot{y}_{E}+c_{33} \cdot \ddot{z}_{E}=\dot{c}_{3}
\end{array}\right. \\
& \left\{\begin{array}{l}
c_{11} \cdot \ddot{x}_{E}+c_{12} \cdot \ddot{y}_{E}+c_{13} \cdot \ddot{z}_{E}= \\
=\dot{c}_{1}-\dot{c}_{11} \cdot \dot{x}_{E}-\dot{c}_{12} \cdot \dot{y}_{E}-\dot{c}_{13} \cdot \dot{z}_{E} \\
c_{21} \cdot \ddot{x}_{E}+c_{22} \cdot \ddot{y}_{E}+c_{23} \cdot \ddot{z}_{E} \\
=\dot{c}_{2}-\dot{c}_{21} \cdot \dot{x}_{E}-\dot{c}_{22} \cdot \dot{y}_{E}-\dot{c}_{23} \cdot \dot{z}_{E} \\
c_{31} \cdot \ddot{x}_{E}+c_{32} \cdot \ddot{y}_{E}+c_{33} \cdot \ddot{z}_{E} \\
=\dot{c}_{3}-\dot{c}_{31} \cdot \dot{x}_{E}-\dot{c}_{32} \cdot \dot{y}_{E}-\dot{c}_{33} \cdot \dot{z}_{E}
\end{array}\right.
\end{aligned}
$$

It identifies the coefficients (92) and the linear system (93) consists of three equations of the first degree each, with three unknowns, which is resolved by the relationships (94):

$$
\begin{aligned}
& \left\{\begin{array}{l}
c_{11}=\alpha, \quad \dot{c}_{11}=\dot{\alpha}, \quad c_{12}=\beta ; \quad \dot{c}_{12}=\dot{\beta} ; \quad c_{13}=\gamma, \quad \dot{c}_{13}=\dot{\gamma}, \\
c_{21}=x_{E}-x_{S} ; \quad \dot{c}_{21}=\dot{x}_{E}-\dot{x}_{S} ; \quad c_{22}=y_{E}-y_{S} ; \quad \dot{c}_{22}=\dot{y}_{E}-\dot{y}_{S} ; \\
c_{23}=z_{E}-z_{S} ; \quad \dot{c}_{23}=\dot{z}_{E}-\dot{z}_{S} ; \quad c_{31}=x_{E}-x_{D} ; \quad \dot{c}_{31}=\dot{x}_{E}-\dot{x}_{D} ; \\
c_{32}=y_{E}-y_{D} ; \quad \dot{c}_{32}=\dot{y}_{E}-\dot{y}_{D} ; \quad c_{33}=z_{E}-z_{D} ; \quad \dot{c}_{33}=\dot{z}_{E}-\dot{z}_{D} ; \\
c_{1}=\alpha \cdot \dot{x}_{S}-\left(x_{E}-x_{S}\right) \cdot \dot{\alpha}+\beta \cdot \dot{y}_{S}-\left(y_{E}-y_{S}\right) \cdot \dot{\beta}+\gamma \cdot \dot{z}_{S}-\left(z_{E}-z_{S}\right) \cdot \dot{\gamma} \\
\dot{c}_{1}=\dot{\alpha} \cdot \dot{x}_{S}+\alpha \cdot \ddot{x}_{S}-\left(\dot{x}_{E}-\dot{x}_{S}\right) \cdot \dot{\alpha}-\left(x_{E}-x_{S}\right) \cdot \ddot{\alpha}+\dot{\beta} \cdot \dot{y}_{S}+\beta \cdot \ddot{y}_{S}- \\
-\left(\dot{y}_{E}-\dot{y}_{S}\right) \cdot \dot{\beta}-\left(y_{E}-y_{S}\right) \cdot \ddot{\beta}+\dot{\gamma} \cdot \dot{z}_{S}+\gamma \cdot \ddot{z}_{S}-\left(\dot{z}_{E}-\dot{z}_{S}\right) \cdot \dot{\gamma}-\left(z_{E}-z_{S}\right) \cdot \ddot{\gamma} \\
c_{2}=\left(x_{E}-x_{S}\right) \cdot \dot{x}_{S}+\left(y_{E}-y_{S}\right) \cdot \dot{y}_{S}+\left(z_{E}-z_{S}\right) \cdot \dot{z}_{S} \\
\dot{c}_{2}=\left(\dot{x}_{E}-\dot{x}_{S}\right) \cdot \dot{x}_{S}+\left(x_{E}-x_{S}\right) \cdot \ddot{x}_{S}+\left(\dot{y}_{E}-\dot{y}_{S}\right) \cdot \dot{y}_{S}+\left(y_{E}-y_{S}\right) \cdot \ddot{y}_{S}+ \\
+\left(\dot{z}_{E}-\dot{z}_{S}\right) \cdot \dot{z}_{S}+\left(z_{E}-z_{S}\right) \cdot \ddot{z}_{S} \\
c_{3}=\left(x_{E}-x_{D}\right) \cdot \dot{x}_{D}+\left(y_{E}-y_{D}\right) \cdot \dot{y}_{D}+\left(z_{E}-z_{D}\right) \cdot \dot{z}_{D} \\
\dot{c}_{3}=\left(\dot{x}_{E}-\dot{x}_{D}\right) \cdot \dot{x}_{D}+\left(x_{E}-x_{D}\right) \cdot \ddot{x}_{D}+\left(\dot{y}_{E}-\dot{y}_{D}\right) \cdot \dot{y}_{D}+\left(y_{E}-y_{D}\right) \cdot \ddot{y}_{D}+ \\
+\left(\dot{z}_{E}-\dot{z}_{D}\right) \cdot \dot{z}_{D}+\left(z_{E}-z_{D}\right) \cdot \ddot{z}_{D} \\
e_{1}=\dot{c}_{1}-\dot{c}_{11} \cdot \dot{x}_{E}-\dot{c}_{12} \cdot \dot{y}_{E}-\dot{c}_{13} \cdot \dot{z}_{E} \\
e_{2}=\dot{c}_{2}-\dot{c}_{21} \cdot \dot{x}_{E}-\dot{c}_{22} \cdot \dot{y}_{E}-\dot{c}_{23} \cdot \dot{z}_{E} \\
e_{3}=\dot{c}_{3}-\dot{c}_{31} \cdot \dot{x}_{E}-\dot{c}_{32} \cdot \dot{y}_{E}-\dot{c}_{33} \cdot \dot{z}_{E}
\end{array}\right. \\
& \left\{\begin{array}{l}
c_{11} \cdot \ddot{x}_{E}+c_{12} \cdot \ddot{y}_{E}+c_{13} \cdot \ddot{z}_{E}=e_{1} \\
c_{21} \cdot \ddot{x}_{E}+c_{22} \cdot \ddot{y}_{E}+c_{23} \cdot \ddot{z}_{E}=e_{2} \\
c_{31} \cdot \ddot{x}_{E}+c_{32} \cdot \ddot{y}_{E}+c_{33} \cdot \ddot{z}_{E}=e_{3}
\end{array}\right.
\end{aligned}
$$

$$
\left\{\begin{array}{l}
\Delta^{(c)}=\left|\begin{array}{lll}
c_{11} & c_{12} & c_{13} \\
c_{21} & c_{22} & c_{23} \\
c_{31} & c_{32} & c_{33}
\end{array}\right|=c_{11} \cdot\left(c_{22} \cdot c_{33}-c_{23} \cdot c_{32}\right) \\
-c_{12} \cdot\left(c_{21} \cdot c_{33}-c_{23} \cdot c_{31}\right)+c_{13} \cdot\left(c_{21} \cdot c_{32}-c_{22} \cdot c_{31}\right) \\
\Delta_{x E 2}=\left|\begin{array}{lll}
e_{1} & c_{12} & c_{13} \\
e_{2} & c_{22} & c_{23} \\
e_{3} & c_{32} & c_{33}
\end{array}\right|=e_{1} \cdot\left(c_{22} \cdot c_{33}-c_{23} \cdot c_{32}\right)- \\
-c_{12} \cdot\left(e_{2} \cdot c_{33}-c_{23} \cdot e_{3}\right)+c_{13} \cdot\left(e_{2} \cdot c_{32}-c_{22} \cdot e_{3}\right) \\
\Delta_{y E 2}=\left|\begin{array}{lll}
c_{11} & e_{1} & c_{13} \\
c_{21} & e_{2} & c_{23} \\
c_{31} & e_{3} & c_{33}
\end{array}\right|=c_{11} \cdot\left(e_{2} \cdot c_{33}-c_{23} \cdot e_{3}\right) \\
-e_{1} \cdot\left(c_{21} \cdot c_{33}-c_{23} \cdot c_{31}\right)+c_{13} \cdot\left(c_{21} \cdot e_{3}-e_{2} \cdot c_{31}\right) \\
\Delta_{z E 2}=\left|\begin{array}{lll}
c_{11} & c_{12} & e_{1} \\
c_{21} & c_{22} & e_{2} \\
c_{31} & c_{32} & e_{3}
\end{array}\right|=c_{11} \cdot\left(c_{22} \cdot e_{3}-e_{2} \cdot c_{32}\right) \\
-c_{12} \cdot\left(c_{21} \cdot e_{3}-e_{2} \cdot c_{31}\right)+e_{1} \cdot\left(c_{21} \cdot c_{32}-c_{22} \cdot c_{31}\right) \\
\ddot{x}_{E}=\frac{\Delta_{x E 2} ; \ddot{y}_{E}=\frac{\Delta_{y E 2}}{\Delta^{(c)}}}{\Delta^{(c)}} ; \quad \ddot{z}_{E}=\frac{\Delta_{z E 2}}{\Delta^{(c)}}
\end{array}\right.
$$

In the following write gear system (95) which is derived by time to obtains the accelerations system (96) which is arranged in the form (97):

$$
\begin{aligned}
& \left\{\begin{array}{l}
d_{11} \cdot \dot{x}_{F}+d_{12} \cdot \dot{y}_{F}+d_{13} \cdot \dot{z}_{F}=d_{1} \\
d_{21} \cdot \dot{x}_{F}+d_{22} \cdot \dot{y}_{F}+d_{23} \cdot \dot{z}_{F}=d_{2} \\
d_{31} \cdot \dot{x}_{F}+d_{32} \cdot \dot{y}_{F}+d_{33} \cdot \dot{z}_{F}=d_{3}
\end{array}\right. \\
& \left\{\begin{array}{l}
\dot{d}_{11} \cdot \dot{x}_{F}+\dot{d}_{12} \cdot \dot{y}_{F}+\dot{d}_{13} \cdot \dot{z}_{F}+d_{11} \cdot \ddot{x}_{F}+ \\
+d_{12} \cdot \ddot{y}_{F}+d_{13} \cdot \ddot{z}_{F}=\dot{d}_{1} \\
\dot{d}_{21} \cdot \dot{x}_{F}+\dot{d}_{22} \cdot \dot{y}_{F}+\dot{d}_{23} \cdot \dot{z}_{F}+d_{21} \cdot \ddot{x}_{F}+ \\
+d_{22} \cdot \ddot{y}_{F}+d_{23} \cdot \ddot{z}_{F}=\dot{d}_{2} \\
\dot{d}_{31} \cdot \dot{x}_{F}+\dot{d}_{32} \cdot \dot{y}_{F}+\dot{d}_{33} \cdot \dot{z}_{F}+d_{31} \cdot \ddot{x}_{F}+ \\
+d_{32} \cdot \ddot{y}_{F}+d_{33} \cdot \ddot{z}_{F}=\dot{d}_{3}
\end{array}\right. \\
& \left\{\begin{array}{l}
d_{11} \cdot \ddot{x}_{F}+d_{12} \cdot \ddot{y}_{F}+d_{13} \cdot \ddot{z}_{F}= \\
=\dot{d}_{1}-\dot{d}_{11} \cdot \dot{x}_{F}-\dot{d}_{12} \cdot \dot{y}_{F}-\dot{d}_{13} \cdot \dot{z}_{F} \\
d_{21} \cdot \ddot{x}_{F}+d_{22} \cdot \ddot{y}_{F}+d_{23} \cdot \ddot{z}_{F}= \\
=\dot{d}_{2}-\dot{d}_{21} \cdot \dot{x}_{F}-\dot{d}_{22} \cdot \dot{y}_{F}-\dot{d}_{23} \cdot \dot{z}_{F} \\
=\dot{d}_{3}-\dot{d}_{31} \cdot \dot{x}_{F}-\dot{d}_{32} \cdot \dot{y}_{F}-\dot{d}_{33} \cdot \dot{z}_{F}
\end{array}\right.
\end{aligned}
$$


The coefficients are determined by relations (98) and the system takes the form (99):

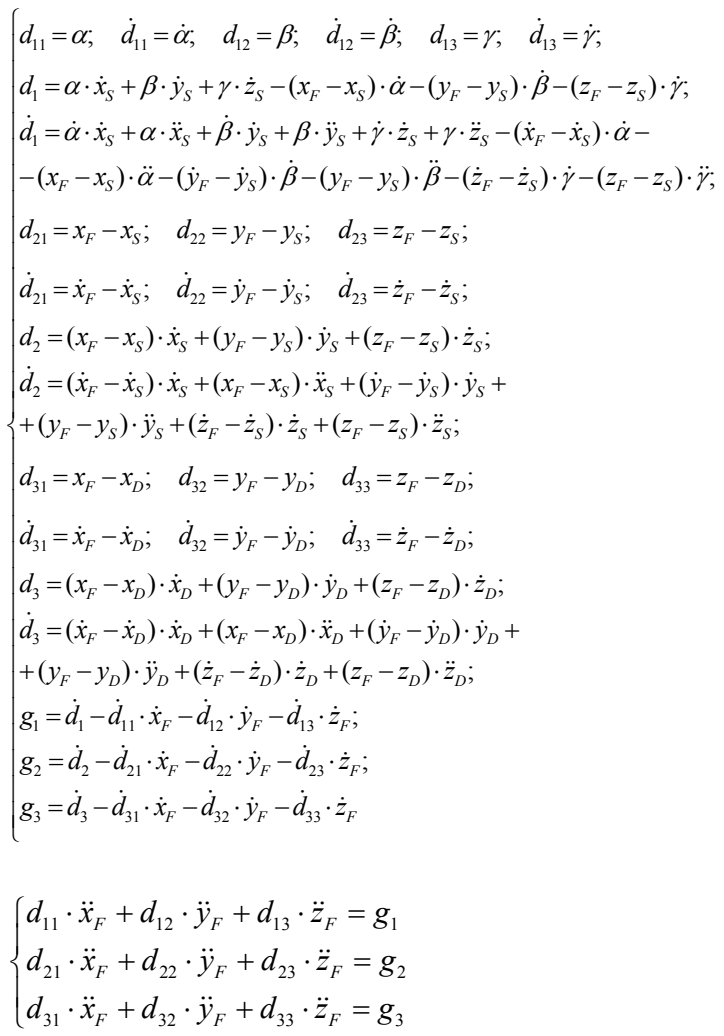

The system (99) with coefficients (98) is solved with the relations (100):

$$
\begin{aligned}
& \left\{\begin{array}{l}
\Delta^{(g)}=\left|\begin{array}{lll}
d_{11} & d_{12} & d_{13} \\
d_{21} & d_{22} & d_{23} \\
d_{31} & d_{32} & d_{33}
\end{array}\right|=d_{11} \cdot\left(d_{22} \cdot d_{33}-d_{23} \cdot d_{32}\right)- \\
-d_{12} \cdot\left(d_{21} \cdot d_{33}-d_{23} \cdot d_{31}\right)+d_{13} \cdot\left(d_{21} \cdot d_{32}-d_{22} \cdot d_{31}\right)
\end{array}\right. \\
& \Delta_{x F 2}=\left|\begin{array}{lll}
g_{1} & d_{12} & d_{13} \\
g_{2} & d_{22} & d_{23} \\
g_{3} & d_{32} & d_{33}
\end{array}\right|=g_{1} \cdot\left(d_{22} \cdot d_{33}-d_{23} \cdot d_{32}\right)- \\
& -d_{12} \cdot\left(g_{2} \cdot d_{33}-d_{23} \cdot g_{3}\right)+d_{13} \cdot\left(g_{2} \cdot d_{32}-d_{22} \cdot g_{3}\right) \\
& \left\{\Delta_{y F 2}=\left|\begin{array}{lll}
d_{11} & g_{1} & d_{13} \\
d_{21} & g_{2} & d_{23} \\
d_{31} & g_{3} & d_{33}
\end{array}\right|=d_{11} \cdot\left(g_{2} \cdot d_{33}-d_{23} \cdot g_{3}\right)-\right. \\
& -g_{1} \cdot\left(d_{21} \cdot d_{33}-d_{23} \cdot d_{31}\right)+d_{13} \cdot\left(d_{21} \cdot g_{3}-g_{2} \cdot d_{31}\right) \\
& \Delta_{z F 2}=\left|\begin{array}{lll}
d_{11} & d_{12} & g_{1} \\
d_{21} & d_{22} & g_{2} \\
d_{31} & d_{32} & g_{3}
\end{array}\right|=d_{11} \cdot\left(d_{22} \cdot g_{3}-g_{2} \cdot d_{32}\right)- \\
& -d_{12} \cdot\left(d_{21} \cdot g_{3}-g_{2} \cdot d_{31}\right)+g_{1} \cdot\left(d_{21} \cdot d_{32}-d_{22} \cdot d_{31}\right) \\
& \ddot{x}_{F}=\frac{\Delta_{x F 2}}{\Delta^{(g)}} ; \quad \ddot{y}_{F}=\frac{\Delta_{y F 2}}{\Delta^{(g)}} ; \quad \ddot{z}_{F}=\frac{\Delta_{z F 2}}{\Delta^{(g)}} ;
\end{aligned}
$$

It writes now the linear velocities system (102) obtained by deriving position system (101). The system (102) derived generates in turn the linear accelerations system (103):

$$
\begin{aligned}
& \left\{\begin{array}{l}
l_{1}^{2}=\left(x_{D}-x_{A}\right)^{2}+\left(y_{D}-y_{A}\right)^{2}+\left(z_{D}-z_{A}\right)^{2} \\
l_{2}^{2}=\left(x_{D}-x_{B}\right)^{2}+\left(y_{D}-y_{B}\right)^{2}+\left(z_{D}-z_{B}\right)^{2} \\
l_{3}^{2}=\left(x_{E}-x_{B}\right)^{2}+\left(y_{E}-y_{B}\right)^{2}+\left(z_{E}-z_{B}\right)^{2} \\
l_{4}^{2}=\left(x_{E}-x_{C}\right)^{2}+\left(y_{E}-y_{C}\right)^{2}+\left(z_{E}-z_{C}\right)^{2} \\
l_{5}^{2}=\left(x_{F}-x_{C}\right)^{2}+\left(y_{F}-y_{C}\right)^{2}+\left(z_{F}-z_{C}\right)^{2} \\
l_{6}^{2}=\left(x_{F}-x_{A}\right)^{2}+\left(y_{F}-y_{A}\right)^{2}+\left(z_{F}-z_{A}\right)^{2}
\end{array}\right. \\
& \left\{\begin{array}{l}
l_{1} \cdot \dot{l}_{1}=\left(x_{D}-x_{A}\right) \cdot \dot{x}_{D}+\left(y_{D}-y_{A}\right) \cdot \dot{y}_{D}+\left(z_{D}-z_{A}\right) \cdot \dot{z}_{D} \\
l_{2} \cdot \dot{l}_{2}=\left(x_{D}-x_{B}\right) \cdot \dot{x}_{D}+\left(y_{D}-y_{B}\right) \cdot \dot{y}_{D}+\left(z_{D}-z_{B}\right) \cdot \dot{z}_{D} \\
l_{3} \cdot \dot{l}_{3}=\left(x_{E}-x_{B}\right) \cdot \dot{x}_{E}+\left(y_{E}-y_{B}\right) \cdot \dot{y}_{E}+\left(z_{E}-z_{B}\right) \cdot \dot{z}_{E} \\
l_{4} \cdot \dot{l}_{4}=\left(x_{E}-x_{C}\right) \cdot \dot{x}_{E}+\left(y_{E}-y_{C}\right) \cdot \dot{y}_{E}+\left(z_{E}-z_{C}\right) \cdot \dot{z}_{E} \\
l_{5} \cdot \dot{l}_{5}=\left(x_{F}-x_{C}\right) \cdot \dot{x}_{F}+\left(y_{F}-y_{C}\right) \cdot \dot{y}_{F}+\left(z_{F}-z_{C}\right) \cdot \dot{z}_{F} \\
l_{6} \cdot \dot{l}_{6}=\left(x_{F}-x_{A}\right) \cdot \dot{x}_{F}+\left(y_{F}-y_{A}\right) \cdot \dot{y}_{F}+\left(z_{F}-z_{A}\right) \cdot \dot{z}_{F}
\end{array}\right.
\end{aligned}
$$

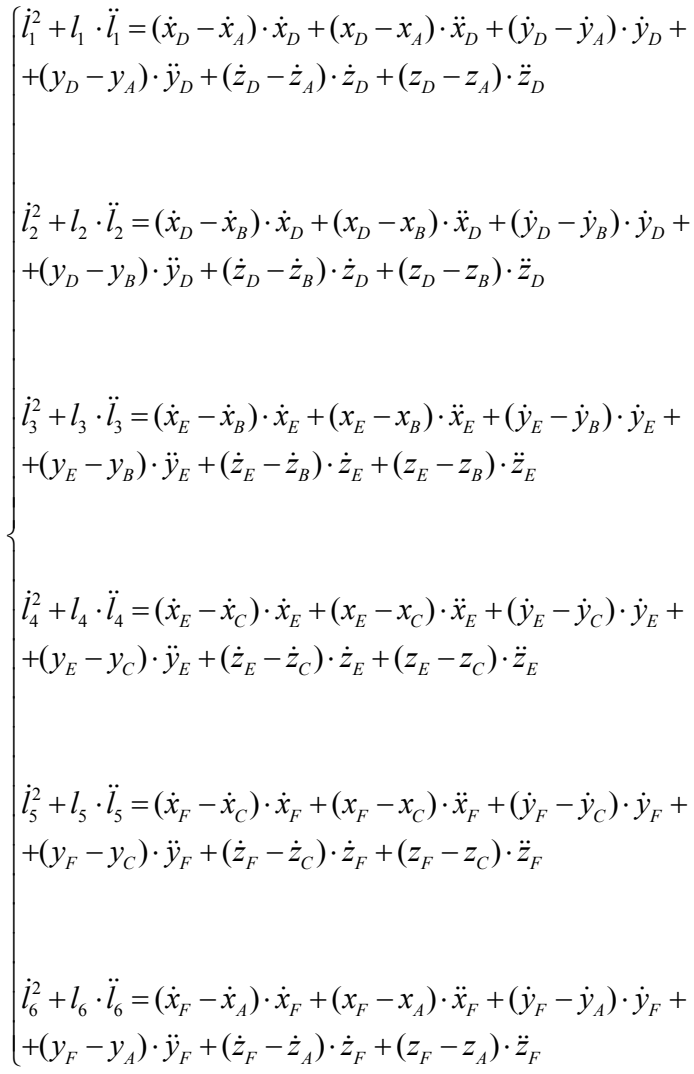


From the system (103) is explicit linear accelerations (104) corresponding to the six feet mobile that supports and acts the superior mobile platform DEF:

$$
\left\{\begin{array}{l}
\ddot{l}_{1}=\left[\left(\dot{x}_{D}-\dot{x}_{A}\right) \cdot \dot{x}_{D}+\left(x_{D}-x_{A}\right) \cdot \ddot{x}_{D}+\left(\dot{y}_{D}-\dot{y}_{A}\right) \cdot \dot{y}_{D}\right. \\
\left.+\left(y_{D}-y_{A}\right) \cdot \ddot{y}_{D}+\left(\dot{z}_{D}-\dot{z}_{A}\right) \cdot \dot{z}_{D}+\left(z_{D}-z_{A}\right) \cdot \ddot{z}_{D}-\dot{l}_{1}^{2}\right] / l_{1} \\
\ddot{l}_{2}=\left[\left(\dot{x}_{D}-\dot{x}_{B}\right) \cdot \dot{x}_{D}+\left(x_{D}-x_{B}\right) \cdot \ddot{x}_{D}+\left(\dot{y}_{D}-\dot{y}_{B}\right) \cdot \dot{y}_{D}\right. \\
\left.+\left(y_{D}-y_{B}\right) \cdot \ddot{y}_{D}+\left(\dot{z}_{D}-\dot{z}_{B}\right) \cdot \dot{z}_{D}+\left(z_{D}-z_{B}\right) \cdot \ddot{z}_{D}-\dot{l}_{2}^{2}\right] / l_{2} \\
\ddot{l_{3}}=\left[\left(\dot{x}_{E}-\dot{x}_{B}\right) \cdot \dot{x}_{E}+\left(x_{E}-x_{B}\right) \cdot \ddot{x}_{E}+\left(\dot{y}_{E}-\dot{y}_{B}\right) \cdot \dot{y}_{E}\right. \\
\left.+\left(y_{E}-y_{B}\right) \cdot \ddot{y}_{E}+\left(\dot{z}_{E}-\dot{z}_{B}\right) \cdot \dot{z}_{E}+\left(z_{E}-z_{B}\right) \cdot \ddot{z}_{E}-\dot{l}_{3}^{2}\right] / l_{3} \\
\ddot{l}_{4}=\left[\left(\dot{x}_{E}-\dot{x}_{C}\right) \cdot \dot{x}_{E}+\left(x_{E}-x_{C}\right) \cdot \ddot{x}_{E}+\left(\dot{y}_{E}-\dot{y}_{C}\right) \cdot \dot{y}_{E}\right. \\
\left.+\left(y_{E}-y_{C}\right) \cdot \ddot{y}_{E}+\left(\dot{z}_{E}-\dot{z}_{C}\right) \cdot \dot{z}_{E}+\left(z_{E}-z_{C}\right) \cdot \ddot{z}_{E}-\dot{l}_{4}^{2}\right] / l_{4} \\
\ddot{l}_{5}=\left[\left(\dot{x}_{F}-\dot{x}_{C}\right) \cdot \dot{x}_{F}+\left(x_{F}-x_{C}\right) \cdot \ddot{x}_{F}+\left(\dot{y}_{F}-\dot{y}_{C}\right) \cdot \dot{y}_{F}\right. \\
\left.+\left(y_{F}-y_{C}\right) \cdot \ddot{y}_{F}+\left(\dot{z}_{F}-\dot{z}_{C}\right) \cdot \dot{z}_{F}+\left(z_{F}-z_{C}\right) \cdot \ddot{z}_{F}-\dot{l}_{5}^{2}\right] / l_{5} \\
\ddot{l}_{6}=\left[\left(\dot{x}_{F}-\dot{x}_{A}\right) \cdot \dot{x}_{F}+\left(x_{F}-x_{A}\right) \cdot \ddot{x}_{F}+\left(\dot{y}_{F}-\dot{y}_{A}\right) \cdot \dot{y}_{F}\right. \\
\left.+\left(y_{F}-y_{A}\right) \cdot \ddot{y}_{F}+\left(\dot{z}_{F}-\dot{z}_{A}\right) \cdot \dot{z}_{F}+\left(z_{F}-z_{A}\right) \cdot \ddot{z}_{F}-\dot{l}_{6}^{2}\right] / l_{6}
\end{array}\right.
$$

\section{Applications}

Presented system can be useful in particular to the surgical robots that operate patients who require an accuracy of positioning very high (Fig. 18).

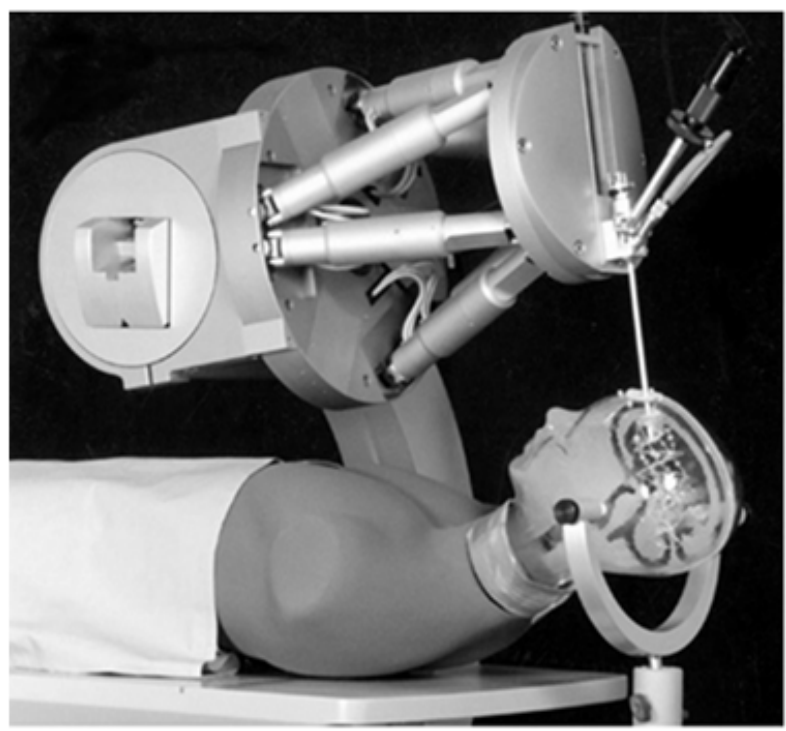

Fig. 18. A surgical robot Stewart system

\section{Results and Discussion}

General surgery is a surgical specialty that focuses primarily on the abdominal cavity organs, such as intestines, then the esophagus, stomach, colon, liver, gall bladder including bile ducts and often also handles the thyroid and hernias.

In Australia, Canada, the USA, the UK and even in Italy, general surgeons also deal with at the level of the breast treatment, including surgery for tumors of the breast itself. In many countries, however, this type of treatment is included in the field of gynecology and obstetrics, in the specialization that Mastology.

\section{Video-Assisted Surgery}

The laparoscopic surgery (VLC), known as laparoscopy (CL), from the greek Lapara, "abdomen", or even as laparoscopy, is a surgical technique that involves the execution of an abdominal surgery without opening of the wall and is a branch of image-guided surgery; It was officially founded in 1987 thanks to Philippe Mouret, surgeon general of Lyon.

In particular, the videoscopia sees, as in endoscopy, an instrument equipped with a camera to a monitor that transmits the images from the inside space examined.

Fundamentals of Endoscopic Surgery

Appendectomy

Removal of Ovarian Cysts

gastric banding

cholecystectomy

laparoscopic jejunostomy

right hemicolectomy

left hemicolectomy

hernioplasty

Gastroenteroanastomosi

Jatoplastica esophageal

nephrectomy

Pancreatectomy body / tail

Plastic hernia

Atypical gastric resection

gastric resection

splenectomy

adrenalectomy

biliary Toilet

Video column

tools container

management of instruments for video-assisted surgery

Open surgery

Surgery Open or Open is the classic mode where you run operations and provide more or less wide opening of the cavities where they reside organs to operate.

Amputation abdominoperineal rectum sec. Miles

Appendectomy

Anal abscess

Removal of Zenker's diverticulum 


\author{
cholecystectomy \\ pancreaticoduodenectomy \\ elastofibroma subscapularis \\ right hemicolectomy \\ left hemicolectomy \\ Milligan Morgan hemorrhoidectomy \\ hepatectomy \\ Hernioplastic groin/femoral/inguinal-scrotal \\ fistulectomy anal \\ Fistulectomy pilonidal sinus \\ total gastrectomy \\ Hysterectomy \\ laparostoma \\ papilloplasty \\ Plastic for abdominal hernia \\ Plastic hernia pericolostomico \\ Placement of Port-a-cath \\ Resection of the rectum via the front \\ Gastric resection \\ recanalization Hartmann \\ saphenectomy \\ splenectomy \\ Thyroidectomy and Paratiroidectomie
}

Thoracic surgery is a branch of surgery that deals with interventions on the lungs and other intrathoracic large formations: The esophagus, lower airways, trachea, bronchi; as well as in case of mediastinal disorders.

It differs from cardiovascular surgery since you do not work your heart. Thoracic surgery interested infinity of malformations, tumors, traumas. Among the most common and studied the medical literature we find, depending on the original site:

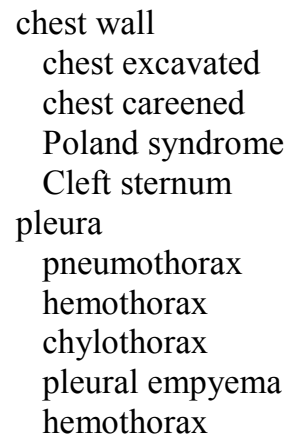

The mediastinum thoracic surgery affects the mediastinum especially for the treatment of different syndromes:

Superior vena cava syndrome

Tracheal compression syndrome

disfagica syndrome

dyspnoeic syndrome

Syndrome Claude-Bernard-Horner

disfonica syndrome

mediastinitis
Struma intrathoracic, typically using the excision of intrathoracic goiter, much more rarely sternotomy, but necessary if there is the presence of a cancer.

pneumomediastinum

thymoma

Trachea The trachea is affected by many diseases: Esophageal-tracheal fistula

Stenosis (are divided into three forms, the generalized hypoplasia, funnel stenosis, segmental stenosis), whose therapy varies depending on the age of the subject, while in adults it operates with stenosis resection in infants where they have not yet completed the development of the trachea you prefer to operate differently behaving endoscopic dilation.

lungs

tumors

bronchogenic cysts

pulmonary sequestration

pulmonary abscess

bullous emphysema

pulmonary tuberculosis

bronchiectasis

Vitreoretinal surgery

anterior vitrectomy

posterior vitrectomy

Avastin intravitreal injection

PR

Constellation

Macular pucker 23/25 G

Removal of silicone oil

Lucentis intravitreal injection

Surgery of the anterior chamber

phacoemulsification micro

standard phacoemulsification

congenital cataracts

Implantation of IOL scleral fixation

trabeculectomy protected

Surgery of the annexes

Recession or muscle Advancement

Recession ++ Faden Desagittalizzazione of Small Oblique

keratoplasty

Affixing of amniotic membrane

Cerclage and Scleral

Dacryocystorhinostomy Ab-internal

Dacryocystorhinostomy Ab-external

Intubation lacrimal

Preparing patients

Anesthesia in Ophthalmology

The general anesthetic is loss of consciousness and pain sensation throughout the body. Induction upon waking you will get the conditions of tranquility and relaxation similar to normal sleep. The loco-regional anesthesia involves the injection of a drug that numbs the area subjected to surgery. Sedation is the 
administration of a medicament that relaxes and calms the patient during surgery. Often sedation is combined locoregional anesthesia.

Index

General notes

Types of Anesthesia

2.1 General Anesthesia

2.2 Loco-regional Anesthesia

2.3 Deep sedation or sedation controlled

2.4 Local anesthesia

pre-anesthetic assessment by the

anesthetist/intensivist

premedication

Post-anesthesia

Criteria for basic intraoperative monitoring

Anesthesia in non-operative environment

Recovery Room

The da Vinci Surgical System (Fig. 2a and 2b) of Intuitive Surgical is a robotic surgery system. It is used most commonly in the removal procedure of the prostate, heart valve replacement and in gynecological surgery procedures, but can also be used for any abdominal or thoracic surgical procedure. It consists of four robotic arms. Three of them are tools that keep objects such as scalpels, scissors, or Bovie electrocautery instruments. The fourth arm carries a camera with two lenses that allows the surgeon a complete stereoscopic vision from the console. The surgeon is sitting at a control panel and look through the crosshairs threedimensional image of the procedure while maneuvering the arms with two pedals and two hand controls.

The da Vinci Surgical System is normally used in the removal of the prostate, heart valve replacement and in gynecological surgery procedures. The da Vinci is an advantage in procedures that focus on a specific area of the abdomen or chest. The interventions that are not localized and which require extensive surgeon's mobility to the different areas are particularly disadvantageous in consideration of the time required for the preparation of the apertures Da Vinci. Surgeons are also experimenting with the da Vinci for the removal of the tumor in the liver and pancreas in the light of the sensitivity of the procedure, the number of blood vessels that the surgeon has to handle and the topical location of the procedure.

It promises that the da Vinci can be used in the future to effect real robotic surgery operations remotely. The possibility of remote operations depends on the availability to the patient of an apparatus da Vinci and someone able to practice the openings, but from a technical point of view the device would allow, for example, to a physician in the United States to operate on a patient in Antarctica.

The advantages of robotic surgery with the da Vinci system are (AB medica): lower margins post-surgical positive (measure indicating residual tumor cells at risk of tumor recurrence)

reduced hospital stay

less postoperative pain

lower risk of infection

less blood loss and fewer transfusions

Smaller scars

better functional recovery of continence and erectile ability in prostate surgery

The da Vinci system by placing the device shared between several hospitals with a surgical transfer of knowhow, can reduce the cost of Robotic Assisted Radical Prostatectomy (RARP), without compromising surgical results, even at the beginning of the learning curve.

Critics of robotic surgery argue that it is difficult to learn the use of the users and there are no studies showing that they are more effective than traditional laparoscopic surgery. The da Vinci system uses proprietary software, which cannot be changed, which limits the freedom of doctors to change the path of the operation In addition, its high cost (about \$ 2 million in the years 2010), makes it prohibitive for many institutions.

The manufacturer, Intuitive Surgical, has been criticized for having achieved a rapid approval by the FDA with a simplified procedure known as "premarket notification" (prior notification to commercialization) that is based on the assumption that the proposed product would be similar to products already previously approved. Intuitive was also accused of providing inadequate training and to encourage health facilities to reduce the number of supervised procedures before a doctor is allowed to use the system without supervision. It is also argued that some patients would have suffered damage due to accidental currents unleashed by inappropriate components of the surgical ends used by the system. Intuitive ribattutto that has the same type of undesirable current can also be originated by nonrobotic laparoscopic procedures. A study published in JAMA-Journal of the American Medical Association claims that the side effects and blood loss in robotic hysterectomies are no better than those obtained by the traditional surgery, despite the much higher cost of the system. In 2013, the FDA began to investigate any issues related to the use of the da Vinci robot, including cases of death during surgery in which the device has been used; are pendants, also, a number of related legal cases to such events.

\section{Milan}

Robotic surgery is a real revolution, to which one can not do without, or a chimera by high costs for the National Health Service? This discussed the major Italian experts, meeting in Forlì for a convention during which they assessed the effectiveness of the technique 
that the expenditure required to buy it and keep it. "The conclusion to which we came-says Domenico Garcea, director of the Surgery Department AUSL Forlì and organizer of the meeting-is that the robot is a definite advantage, for which you pay a substantial figure. Several studies and Italian experiences have shown, however, that costs you can break down if it is a multi-disciplinary use, with a targeted selection of patients to operate and proper training of surgeons and all medical personnel. "The price of the last model is around two million and 400 thousand euro and today there are about 50 hospitals, most of them public, who bought a robot. Some properties, such as the European Institute of Oncology and the San Raffaele in Milan, after careful medical and economic assessments have decided to buy a second. The hospital of Grosseto, then, has already taken the third.

\section{The Pros and Cons of the Robot I}

"The benefits for patients-continues Garcea-are the same that can be achieved with the minimally invasive surgery: Smaller scars, fewer complications, shorter hospital stays, improved recovery times. The fact is that the robot gives the surgeon the ability to move, to turn a hand or an arm, 360 degrees: Something humanly impossible for a human being. Or to arrive at a precise point without doing contortions strenuous, take tough positions to hold for hours. It also gives us a threedimensional vision, with a visual and a perfect light. unfavorable aspects for the sick, compared to traditional surgery, there are none. "The cons, however, exist for the hospital's coffers or the NHS: To date, when it comes to robotic surgery we are a monopoly. The only available specimen is the Da Vinci, it arrived on the market at the end of the nineties and then updated in five models. As long as the patent expires there is no competition, even if "now, the robot purchase is almost never be borne by the health authorities, but it is often the result of a donation by private entities-comments Andrea Coratti, director of general surgery hospital of Grosseto Misericordia and pioneer, along with Pier Cristoforo Giulianotti, the Italian robotic surgery. They are mainly operating costs that should be cut." (AB medica).

\section{Possible Solutions to Reduce Costs}

The robot therefore, at least for now, involves too high costs because many hospitals can afford to buy one? Not according Franca Melfi, an expert in thoracic robotic surgery, which contains data a study sponsored by the Thoracic Surgery, University of Pisa: "If you use a machine for a number of applications per year, identified in about 300, surgery robotics is not only expensive, but saves resources-explains. Based on this principle, the Tuscany Region has approved a project to expand the robotics "economically compatible." In practice, the Region has allocated funds for the purchase of new robots, which will be used, however, in the interdepartmental way: Physically placed in some facilities will be used in turn by most surgeons to more hospitals. "The principle, therefore, is to depreciate the investment using the Da Vinci for a large number of applications every year, for different diseases and in patients specially selected. "These devices today can and must have a variety of applications-precise Giulianotti, started from Grosseto and came three years ago in Chicago, where he directs the Department of General Surgery and robotics at the University. For example in urology, gynecology, cardio-thoracic and gastrointestinal surgery, pediatrics or oncology. In the coming years, then, the robot can always use more in the cervical region, thyroid, in otorhinolaryngology and the major operations of the colon, liver, pancreas and kidneys."

\section{Reserved for 'Formula one Drivers'}

The conclusions of the conference emerges a final rule: To use the robot serves a clear and sufficient training. The greater the specialization of the surgeon, the better is the way to use the equipment. "It's a little 'how to drive a car in a race of Formula One: We are not all capable and, in any case, to be good pilots must prepare a lot and constantly updated" exemplifies Garcea. "You have to buy dexterity and, as in any field, one learns and improves with practice-explains Paolo Bianchi, Unit Director of the European Institute of Oncology minimally invasive surgery of Milan and founding member of the Clinical robotic surgical association -. In this way not only we ensure the best outcome for patients, but-once againrestrict the costs (of wear, expensive disposable equipment related apparatus, time). The robot, in conclusion, is a tool that essentially facilitates the surgeon and makes it possible to perform minimally invasive treatments that more and more complex, with traditional laparoscopic technique, only a few surgeons would be very long and practicable many experts", (Chirurgia robotica, la rivoluzione in medicina).

Very few thousand worldwide, fifty in Italy. It is of very high precision robotic equipment and technology that assist surgeons in the most complex and important treatments.

Integrated systems of video-shooting, laser, mechanical arts help the surgeon to realize interventions otherwise almost impossible, such as the removal of tumors defined until a short time ago inoperable, or by the very high cost, both in economic terms-think the cost of a prolonged hospital stay by an invasive intervention-both as endurance of physical pain for patients (Chirurgia robotica, la fantascienza incontra la medicina).

Of all these tools we talk during the VII European Congress of laparoscopic and robotic surgery going on these days in Rome. More than 400 doctors have come from all over the world, who will attend to some of the most complex operations. 
You can ask a question to the forum specialists or complexes made at the clinic Pius XI in Rome, explains Vito Pansadoro, surgeon urologist and president of Congress, "is the first time ever that an international meeting is designed to bring the laparoscopy and robotics to its extremes. Are all cases the operability limits, that only thanks to surgeons of the highest caliber have become possible now $60 \%$ of worldwide operations performed with the Da Vinci robot-explains Pansadororelate to prostate cancer, in the United States are $186 \%$, followed by the removal of kidney tumors, cystectomies and stenosis of the urinary tract".

Highs are the costs of a surgeon robot, about two million dollars, in addition to $\$ 150,000$ a year for maintenance. However, in some cases it is indispensable: "We have solved cases with a narrowing of the urinary tract due to endometriosis or patients operated without success already four or five times," says Prof. yet. Pansadoro. There are many efforts in areas of the body difficult to reach; such as replacing orthotopic bladder, lymph node dissection and tumors of the testis, the partial or total nephrectomy, "in which laparoscopy becomes necessary when we have only one kidney," recalls Pansadoro. With regard to kidney surgery, the professor adds: "Today $60-70 \%$ of kidney tumors are discovered by chance through an ultrasound or a CT performed for other purposes and the possibility of intervening with ever less invasive capacity represents the next surgery".

The latest invention in terms of robotics applied to medicine is the "fetus-robots." Designed by researchers University of Tokyo, he is able to simulate the movements of the fetus in the amniotic fluid. The goal is to study the child's coordination skills, treat early disease of the newborn and improve intrauterine and neonatal surgery. If Japanese MPRE is the robot-nurse prototype, a humanoid upper $155 \mathrm{~cm}$ and $70 \mathrm{~kg}$ of weight. The automaton is able to collect, move and transport small or large objects, up to a weight of 30 kilograms. The robot may soon be used in hospital wards for the transport of materials, drugs and patients.

Finally, the dream to travel inside the human body has already become reality. Israeli scientists have invented a pill equipped with a miniature camera, once swallowed, travels and films him in the gut, avoiding the introduction of probes for diagnostic purposes such as gastro-scopy and colonoscopy (MEDICINA ROBOTICA).

\section{Conclusion}

Presented mechatronic systems, are the most advanced robotic systems. They have a spatial operation with many degrees of freedom. For this reason are still difficult to program. Two issues must be solved in order that they may be programmed easily and accurately. First problem engineering judgment is to determine the coordinates of entry into depending on the coordinates of the required at the outlet. This problem is solved precisely by systems of the original equations presented within the framework of the work. The second issue that appears in these systems mechatronic and parallel, is the movement and their positioning with a precision very high. The following equations can solve the accuracy required but is required and a high precision of the systems of actuator. Fortunately today, actuator (linear actuator) problem of high precision has already been solved. Today we have linear actuator of high precision, pneumatic and or electric. Precision linear actuators are designed to deliver high performance in applications requiring continuous duty operation. They provide the highest thrust and speed of any electric actuator and allow you to design more compact machinery with maximum throughput. Often chosen as a clean and efficient alternative to fluid power solutions, precision linear actuators draw energy only on demand and operate more quietly than pneumatics. The system presented in the work is a clear example of involvement of engineering in medical problems.

\section{Acknowledgement}

This text was acknowledged and appreciated by Assoc. Pro. Taher M. Abu-Lebdeh, North Carolina A and T State Univesity, United States, Muftah H. El-Naas PhD MCIC FICCE QAFCO Chair Professor in Chemical Process Engineering Gas Processing Center College of Engineering Qatar University and (Ms.) Shweta Agarwala Senior Research Scientist at Singapore Center for 3D Printing Nanyang Technological University Singapore, whom we thanks and in this way.

\section{Funding Information}

This research has been funded by Italian Ministry of University and Research project FIRB Future in Research 2008 project RBFR08T83J.

\section{Author's Contributions}

All the authors contributed equally to prepare, develop and carry out this work.

\section{Ethics}

This article is original. Author declares that are not ethical issues that may arise after the publication of this manuscript.

\section{References}

AB medica.
http://www.abmedica.it/it/robotica 
Akubue, A., 2011. Appropriate technology for socioeconomic development in third world countries. J. Technol. Stud., 26: 33-43. DOI: $10.21061 /$ jots.v26i1.a.6

Beretta, G., 2014. Sexual Problems and Infertility. In: Clinical Management of Male Infertility, Cavallini, G. and G. Beretta (Eds.), Springer International Publishing, Switzerland.

Cao, W., H. Ding, Z.I. Bin and C. Ziming, 2013. New structural representation and digital-analysis platform for symmetrical parallel mechanisms. Int. J. Adv. Robotic Syst., 10: 243-243.

Chirurgia robotica, Da Wikipedia, l'enciclopedia libera. https://it.wikipedia.org/wiki/Chirurgia_robotica

Chirurgia robotica, la rivoluzione in medicina. http://www.corriere.it/salute/sportello_cancro/11_gi ugno_27/chirurgia-robotica-davincimartinella_b11fe5fa-2f97-11e0-a47400144f02aabc.shtml?refresh_ce-cp

Chirurgia robotica, la fantascienza incontra la medicina. http://www.italiasalute.it/news.asp?ID=4491

Chiu, S., K. Joseph, S. Ghosh, R.M. Cornand and D. Schiller, 2015. Reasons for delays in diagnosis of anal cancer and the effect on patient satisfaction. Can. Fam. Physician, 61: e509-e516. PMID: 26889506

Da Vinci Surgical System, From Wikipedia, the free. https://en.wikipedia.org/wiki/Da_Vinci_Surgical_Sy stem

DRE: Its importance in the disciplines uro-andrological. http://www.medicitalia.it/minforma/urologia/2310esplorazione-rettale-importanza-discipline-uroandrologiche.html

Garcia, M.M., A.J. Gallardo and C.E. Castillo, 2013. Finding the generalized forces of a series-parallel manipulator. Int. J. Adv. Robotic Syst., 10: 4-4. DOI: $10.5772 / 53824$

Little, P., M. Dorward, G. Warner, K. Stephens and J. Senior et al., 2004. Importance of patient pressure and perceived pressure and perceived medical need for investigations, referral and prescribing in primary care: Nested observational study. BMJ, 328: 444-6. DOI: $10.1136 / \mathrm{bmj} .38013 .644086 .7 \mathrm{C}$
MEDICINA

ROBOTICA.

http://online.scuola.zanichelli.it/fare/files/2008/09/6 685 paci_fare_medicina_robotica.pdf

Nickel, J.C., 2010. Guidelines for the management of benign prostatic hyperplasia. Can. Urol. Assoc. J., 4: 310-316.

Petrescu, F.I. and R.V. Petrescu, 2013. Cinematics of the 3R Dyad. J. Engevista, 15: 118-124.

Petrescu, F.I. and R.V. Petrescu, 2012. MecatronicaSisteme Seriale si Paralele. 1st Edn., Create Space Publisher, USA, ISBN-10: 1475066139, pp: 128.

Petrescu, F.I and R.V. Petrescu, 2011. Mechanical Systems, Serial and Parallel. 1st Edn., Lulu.com, ISBN-10: 1446600394, pp: 124.

Petrescu, F.I., B. Grecu, A. Comanescu and R.V. Petrescu, 2009. Some mechanical design elements. Proceedings of the 3rd International Conference on Computational Mechanics and Virtual Engineering, (MVE' 09), Braşov, pp: 520-525.

Reilly, B.M., 2003. Physical examination in the care of medical inpatients: an observational study. Lancet. 362: 1100-5. PMID: 14550696

Seehusen, D.A., 2007. Pelvic examination. N Engl. J. Med., 357: 1778-9.

Zanollo, A., G. Beretta and L. Zanollo, 1991. Anamnestic criteria for sexual evaluation in the andrological field. Arch Ital Urol Nefrol. Androl., 63: 493-7. PMID: 1838838

Walsh, A.L., 2014. Digital rectal examination in primary care is important for early detection of prostate cancer: A retrospective cohort analysis study. Br J. Gen. Pract., 64: e783-7. DOI: 10.3399/bjgp14X682861

Wang, K., M. Luo, T. Mei, J. Zhao and Y. Cao, 2013. Dynamics analysis of a three-DOF planar serialparallel mechanism for active dynamic balancing with respect to a given trajectory. Int. J. Adv. Robotic Syst., 10: 23-23. DOI: 10.5772/54201 